\title{
Buoyancy-Driven Chaotic Regimes During Solute Dispersion in Pore Networks
}

\author{
C.D. Tsakiroglou ${ }^{*}$, M.A. Theodoropoulou ${ }^{1}$ and V. Karoutsos ${ }^{1}$ \\ 1 Institute of Chemical Engineering and High Temperature Chemical Processes, Foundation for Research and Technology Hellas, \\ Stadiou Str., Platani, PO Box 1414, 26504 Patras - Greece \\ e-mail: ctsakir@iceht.forth.gr - mtheod@iceht.forth.gr - vkar@iceht.forth.gr \\ * Corresponding author
}

\begin{abstract}
Résumé - Régimes chaotiques dans la dispersion de solutés sous contrôle gravitaire, en réseaux poreux modèles - Le souci d'examiner les effets gravitaires sur la dispersion de solutés à l'échelle d'un réseau poreux conduit à réaliser des expériences de transport de soluté à partir d'une source unique, au sein de réseaux confectionnés dans du verre. Ces réseaux ont des morphologies et des degrés d'hétérogénéité, à l'échelle du pore, qui sont variés. Leur conception permet une visualisation des expériences de transport. Une solution aqueuse faiblement concentrée s'écoule en régime permanent à travers le réseau, et une solution plus concentrée en soluté (et donc relativement plus dense) est injectée à débit constant et très faible, au moyen d'une buse disposée à l'intérieur du système. L'évolution en régime transitoire des concentrations, en diverses zones du milieu poreux, est enregistrée à diverses échelles par un dispositif vidéo et un analyseur qui transforme des intensités lumineuses en concentrations. En l'absence d'effet gravitaire, le régime permanent de dispersion varie avec le nombre de Péclet $(\mathrm{Pe})$. Les dispersivités longitudinale et transversale sont estimées en calant des solutions analytiques approchées de l'équation d'advection-dispersion sur les points expérimentaux. Sous l'effet de la gravité, de multiples régimes permanents de dispersion peuvent s'établir pour chaque valeur de $P e$, et des lobes instables de concentration en soluté sont observés à mesure que le flux descendant de solution plus dense est contrebalancé par le flux ascendant de liquide plus léger. Les régimes en question peuvent être périodiques, quasi périodiques, ou chaotiques, selon la valeur des paramètres caractéristiques du sytème. La nature des fluctuations transitoires autour de la concentration moyenne en soluté est analysée en mesurant comment leur période est distribuée, et en identifiant les fréquences fondamentales du spectre de puissance après transformation de Fourier. On s'aperçoit que les zones de mélange tendent à se stabiliser lorsque $P e$ augmente, et que les régimes périodiques ou quasi périodiques sont favorisés aux fortes valeurs de $P e$ et aux faibles niveaux d'hétérogénéité à l'échelle du pore, tandis que l'inverse est observé pour les régimes chaotiques.
\end{abstract}

\footnotetext{
Abstract - Buoyancy-Driven Chaotic Regimes During Solute Dispersion in Pore Networks - In an attempt to investigate gravity effects on solute dispersion at the scale of a pore network, single sourcesolute transport visualization experiments are performed on glass-etched pore networks of varying morphology and degree of pore-scale heterogeneities. The (lighter) low solute concentration aqueous solution flows steadily through the porous medium and the (heavier) high solute concentration solution is injected at a very low and constant flow rate through an inner port. The transient evolution of the solute concentration distribution over various regions of the pore network is determined at different scales by capturing and video-recording snapshots of the dispersion on PC, measuring automatically the spatial
} 
variation of the color intensity of the solution, and transforming the color intensities to solute concentrations. Without the action of gravity, the steady-state dispersion regime changes with Peclet $(P e)$ number, and the longitudinal and transverse dispersivities are estimated by fitting the experimental datasets to approximate analytic solutions of the advection-dispersion equation. Under the action of gravity, multiple of steady-state solute dispersion regimes is developed at each Pe value, and lobe-shaped instabilities of the solute concentration are observed across the pore network, as the downward flow of the denser (higher solute concentration) fluid is counterbalanced by the upward flow of the less dense (lower solute concentration) fluid. The steady-state dispersion regimes may be periodic, quasi-periodic or chaotic depending on the system parameters. The nature of the transient fluctuations of the average solute concentration is analyzed by identifying the periodicity of the fluctuations, determining the autocorrelation function and the statistical moments of the time series, and inspecting the FFT (fast Fourier transform) power spectra. It is found that the mixing zone tends to be stabilized at higher values of the Peclet $(\mathrm{Pe})$ number. Periodic and quasi-periodic solute dispersion regimes are favored by relatively high Pe values and low degree of pore scale heterogeneities, whereas chaotic regimes are favored by low Pe values and high degree of pore-scale heterogeneities. Some ambiguity concerning the classification of the observed solute dispersion regimes is due to the fact that the short length of the time series does not allow the processing of datasets with the nonlinear methods of state-space analysis.

\section{NOTATION}

$a_{L} \quad$ longitudinal dispersivity

$a_{T} \quad$ transverse dispersivity

$b \quad$ diameter of solute source

C solute concentration

$C_{0} \quad$ solute concentration in feed solution

$C_{i} \quad$ solute concentration in source solution

D dispersion tensor

$D_{\text {eff }}$ solute/solvent effective diffusion coefficient in the porous medium

$D_{i j} \quad$ component of dispersion tensor

$D_{m} \quad$ solute/solvent molecular diffusion coefficient

$D_{L} \quad$ longitudinal dispersion coefficient

$D_{T} \quad$ transverse dispersion coefficient

$d_{p} \quad$ pore depth

$d_{s} \quad$ distance of solute source from the upper lateral boundary

$F \quad$ flatness of the signal probability distribution

$f \quad$ scalar function of the spatial variation of permeability

$F_{f} \quad$ formation factor

$F_{m} \quad$ solute flux

g gravity acceleration vector

$k \quad$ porous medium permeability

$l_{p} \quad$ pore length

$\stackrel{p}{P} \quad$ fluid pressure

$P e \quad$ Peclet number

$R a \quad$ Rayleigh number

$R a_{1} \quad$ Rayleigh number for $\beta\left(C_{i}-C_{0}\right)=1.0$

$R a_{m} \quad$ modified Rayleigh number

$S \quad$ skewness of the signal probability distribution

$t$ time

u pore (intrinsic) flow velocity vector

$u_{i} \quad$ component of flow velocity along $i$ direction $u_{0} \quad$ asymptotic constant pore velocity

$W \quad$ width of porous medium

$w_{p} \quad$ pore width

$x$ horizontal coordinate

$y \quad$ vertical coordinate

$z \quad$ transient signal

$\langle z\rangle \quad$ time averaged value of the signal.

\section{Greek Letters}

$\beta \quad$ coefficient of (mass) volumetric expansion

$\Delta \tau \quad$ dimensionless time difference

$\zeta$ dimensionless vertical coordinate

$\mu \quad$ solution viscosity

$\xi$ dimensionless horizontal coordinate

$\rho \quad$ solution density

$\rho_{0} \quad$ solution density at concentration $C_{0}$

$\sigma_{d} \quad$ standard deviation of the pore depth distribution

$\sigma_{w} \quad$ standard deviation of the pore width distribution

$\sigma_{\tau d} \quad$ standard deviation of the distribution of the periods of solute concentration fluctuations

$\tau$ dimensionless time

$\tau_{d} \quad$ dimensionless period of solute concentration fluctuations

$\phi \quad$ volumetric porosity

$\phi_{a} \quad$ areal porosity

$\psi \quad$ stream function.

\section{Symbols}

$\nabla \quad$ gradient vector

$\nabla$. divergence

* dimensionless variable

\langle\rangle$\quad$ mean value. 


\section{INTRODUCTION}

Over the past years, there has been considerable interest to study the migration of solute in groundwater. Many of the ongoing environmental problems in groundwater involve issues including groundwater contamination, seawater intrusion in coastal aquifers, radioactive waste disposal, geothermal energy development, groundwater-surface water interactions, and subsurface storage of materials, fluids and energy (Simmons et al., 2001).

Field and laboratory studies have shown that fluid density gradients caused by variations in solute concentration and/or temperature can play an important role in solute transport in groundwater (Liu and Dane, 1997; Simmons and Narayan, 1997; van Duijn et al., 1998). When the density of the invading plume is greater than that of the ambient groundwater, density driven flow may result in lobe-shaped instabilities and fingering. Density stratification, where a dense fluid overlies a less dense one, is the main reason of such instabilities (free convection), which have been studied for almost a century (Turner, 1973; Drazin, 2002). In essence, the buoyancy-driven free convection enhances the hydrodynamic mixing of plume with groundwater by decreasing the time-scales of mixing, increasing both the total quantity of transferred solute, and the dimensions of the mixing zone (Schincariol, 1998).

In several cases, dense reactive and non-reactive solutes may migrate through groundwater. Examples are:

- uncontrolled landfills;

- saltwater intrusion;

- toxic chemical disposal sites;

- $\mathrm{CO}_{2}$ storage in aquifers.

In these cases, instabilities may arise from the coupling of chemical reactions with density-driven convective flow (Freedman and Ibaraki, 2002). Results show that the instability development is sensitive to the initial perturbation caused by the density difference between the solute plume and groundwater. If the initial perturbation is quite large then it acts as a "trigger" in the flow system leading to the development of instabilities across a planar reaction front. When permeability changes occur, due to dissolution reactions, a density-dependent reactive feedback loop may be created (Freedman and Ibaraki, 2002). In real porous media, different styles of heterogeneity may be considered ranging from continuously trending heterogeneity (where the permeability fluctuates randomly at the scale of investigation) to discretely fractured geologic media. Results indicate that the onset of instabilities and their subsequent growth and decay are related to the structure and variance of the permeability field (Schincariol, 1998).

Most studies investigating buoyancy driven instabilities in solute dispersion have been focused on transient (Simmons et al., 2001) or free convective flows (Schoofs et al., 1999), while multiple steady-state dispersion regimes that may arise from the coupling of diffusion with forced and free convective flows have been overlooked. The study of asymptotic chaotic steady-state regimes is associated with points of multiple bifurcation (Drazin, 2002). This is the situation which occurs when a stable equilibrium loses stability and transits to a new asymptotic state with a complicated dynamics. The most classical problem belonging to this category is the thermohaline convection in a horizontal layer of incompressible fluid having fixed temperatures and salt concentrations on its upper and lower boundaries (Turner, 1973; Proctor and Weiss, 1990; Vadasz, 1999). The routes to chaos in a fluid saturated porous layer heated from below (Schoofs et al., 1999; Bera and Khalili, 2002) along with the nature of chaotic flows induced by thermo-chemical convection (Basu and Islam, 1996) have been studied theoretically. In spite of the numerous efforts that have been done to simulate numerically density-driven instabilities (Diersch and Kolditz, 2002), a few experimental datasets are available and most of them refer to large-scale systems where the pore scale phenomena are overlooked.

In the present study, solute dispersion visualization experiments are performed on glass-etched pore networks where the gravity acts perpendicular to the main flow direction of a low solute concentration solution. A high solute concentration solution is injected at low flow rate through a hole (Fig. 2, single source). The buoyancy-driven multiple steady-state solute dispersion regimes which are observed in three model pore networks of different morphology, are identified and quantified at various Peclet numbers. The periodic, quasi-periodic or chaotic nature of the transient fluctuations of the average solute concentration of a small region of the pore network is examined by analyzing the dataset in both time and frequency FFT (Fast Fourier Transform) domains.

\section{MATERIALS AND METHODS}

Photolithography was used to fabricate artificial large 2D pore networks (Tsakiroglou et al., 2003) by etching mirror image patterns (Fig. 1) on two glass plates with hydrofluoric acid, and sintering the prealigned etched plates in a programmable muffle furnace (the sintering is carried out by increasing the temperature in a stepwise fashion up to a maximum value of $650^{\circ} \mathrm{C}$ ). The selected patterns of pore networks (Fig. 1) represent homogeneous (M-1, S-1) and heterogeneous (D-1) porous media having high (M-1, D-1) or low (S-1, D-1) pore density (porosity), and low (M-1), moderate (S-1) and high (D-1) variability of the pore sizes (Table 1). The pore cross-sectional shape in these models is lenticular (Tsakiroglou et al., 1997). The pore width distribution $\left(\left\langle w_{p}\right\rangle, \sigma_{w}\right)$ was determined by estimating the relative variation caused on the pore sizes of the patterns (Fig. 1) by the etching and sintering processes, whereas the pore depth 
TABLE 1

Parameters of model pore networks

\begin{tabular}{l|c|c|c|c}
\hline Topology & $\begin{array}{c}\text { Simple net - } \\
\text { Square lattice } \\
\text { Model M-1 }\end{array}$ & $\begin{array}{c}\text { Simple net - } \\
\text { Square lattice } \\
\text { Model S-1 }\end{array}$ & \multicolumn{2}{|c}{$\begin{array}{c}\text { Dual network -Square lattice } \\
\text { Large pores }\end{array}$} \\
Model D-1
\end{tabular}

distribution $\left(\left\langle d_{p}, \sigma_{d}\right\rangle\right)$ was estimated by matching the calculated value of the absolute permeability to the experimentally measured one (Table 1). The model pore network D-1 originates from the superposition of the network $\mathrm{S}-1$ on a dense network of thin and short pores of identical size (Table 1 , Fig. 1). For the calculation of the permeability, a hypothetical cross-sectional area equal to $W l_{p}$ was used in Darcy law. In this manner, although the models S-1 and D-1 have larger pore dimensions than those of model M-1, their permeabilities appear smaller than that of model M-1 (Table 1).

An experimental apparatus was constructed to perform visualization experiments of multiphase flow on planar pore networks (Theodoropoulou et al., 2003). The entire system was placed in a cooled incubator (Fig. 2a) in order to maintain the temperature constant and to avoid any undesired changes in fluid properties. Details about the type of fluids used in experiments are reported elsewhere (Theodoropoulou et al., 2003). The fluids were injected into the pore network through four inlet ports and expelled from it through four outlet ports. In addition, four holes were drilled on the centre of the network that served either as solute sources or pressure taps (Fig. 2b). Two syringe pumps were used for the injection of fluids. A CCD video-camera, connected to an image grabber built-in PC, was used to capture images and store them directly on the hard-disk of the PC (Fig. 2a).

A technique was developed to measure the transient changes of the solute concentration distribution throughout the pore network (Theodoropoulou et al., 2003). The technique is based on the detection of the color changes caused by the mixing of a dilute $\mathrm{HCl}\left(C_{0}=0.03 \mathrm{kmol} / \mathrm{m}^{3}\right)$ with a dense $\mathrm{HCl}\left(C_{i}=0.41 \mathrm{kmol} / \mathrm{m}^{3}\right)$ solution. A mixture of organic color indicators was selected after testing the sensitivity of the color variation with $\mathrm{pH}$ (Theodoropoulou et al., 2003). As the $\mathrm{pH}$ varies in the range 1-3, structural changes caused on the chromophore groups of indicators affect drastically the absorption intensity of each colour. The
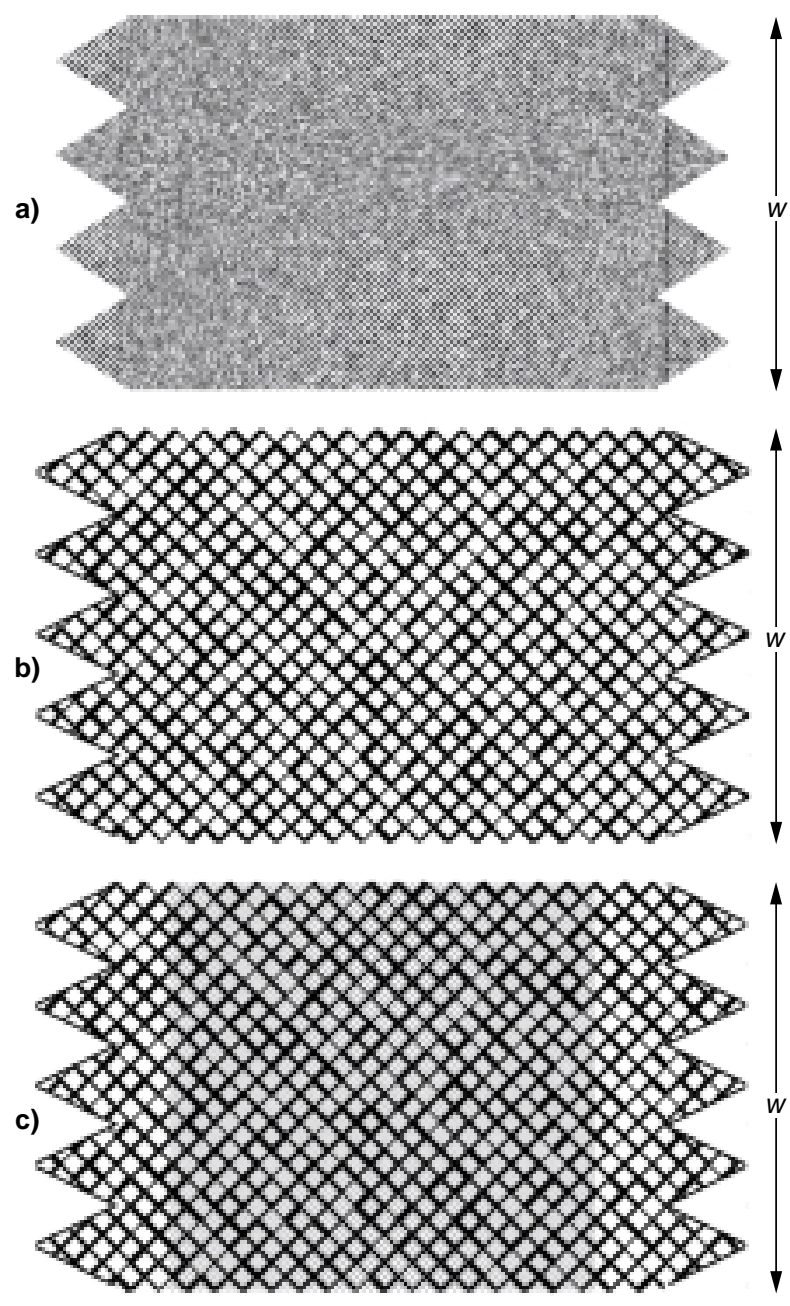

Figure 1

Patterns of the three model pore networks (see Table 1): a) M-1 $(W=0.104 \mathrm{~m}$; b) S-1 $(W=0.113 \mathrm{~m})$; c) D-1 $(W=0.113 \mathrm{~m})$. 
a)

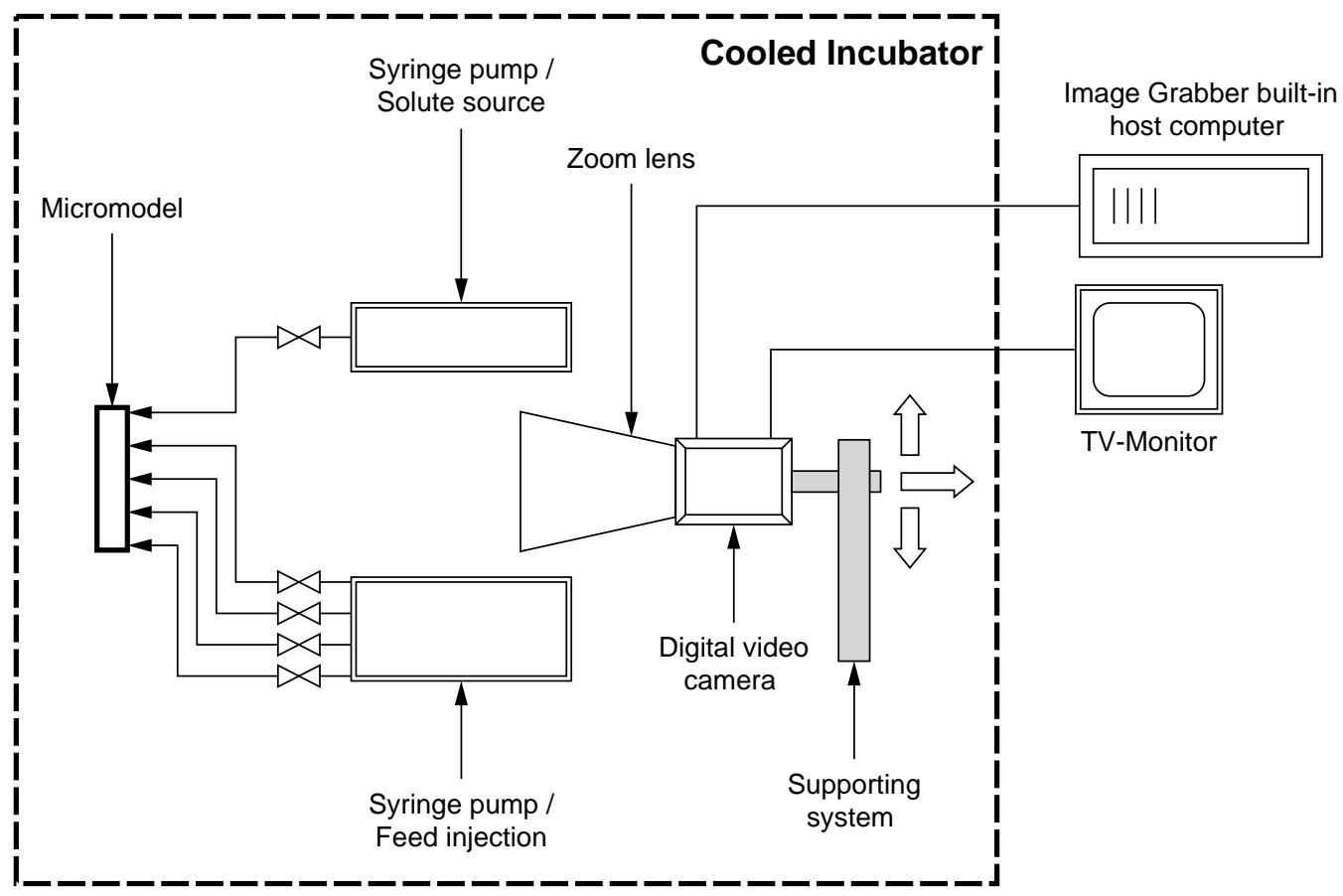

b)

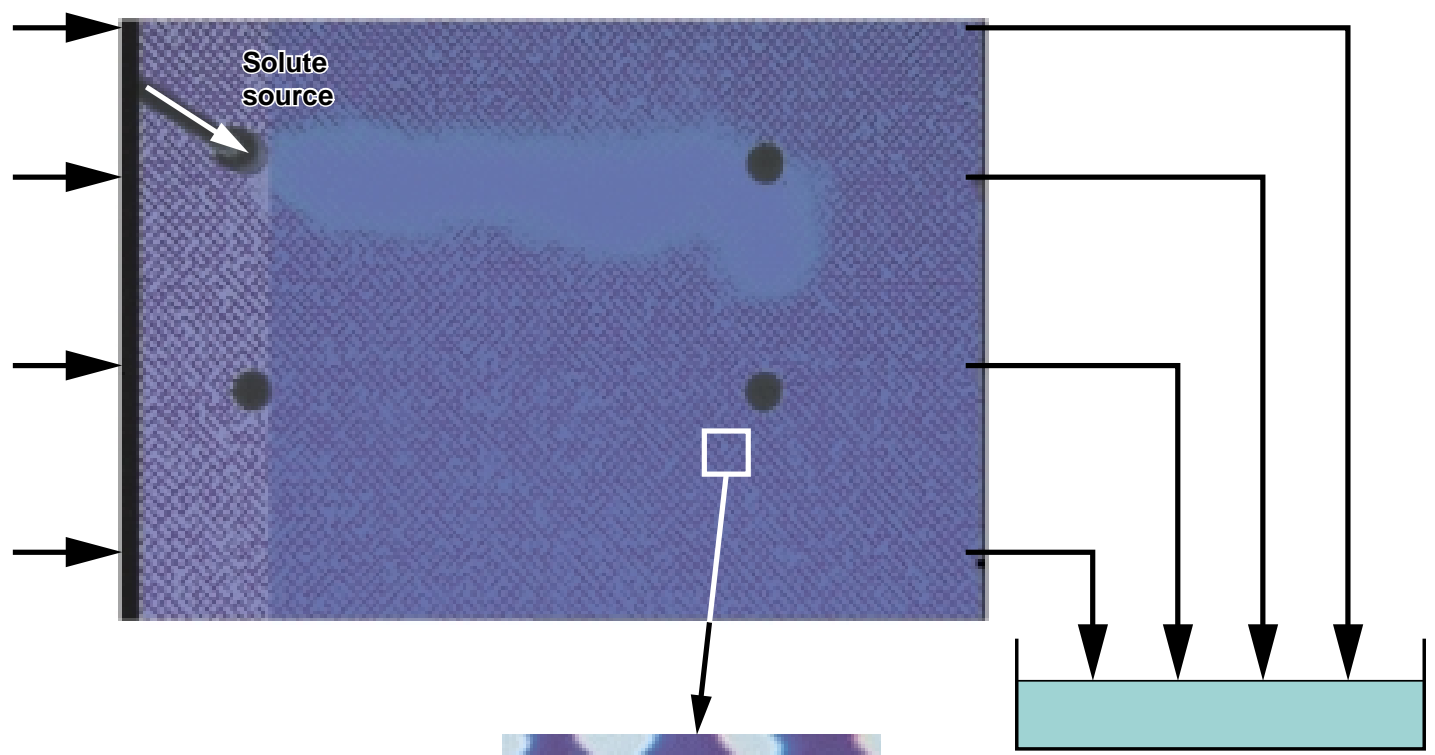

c)

$$
w_{p} \longmapsto
$$$$
\text { Unit }
$$$$
\text { cell }
$$

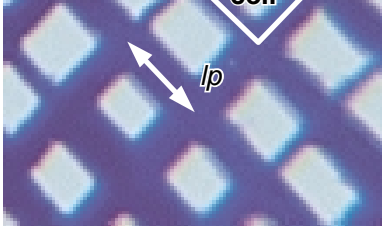

Figure 2

a) schematic diagram of the experimental set-up; b) detailed representation of the flow conditions in model pore network (the lighter the colour the higher the solute concentration); c) pore width and length. 
color of indicators, contained in traces in both solutions, was so sensitive to the $\mathrm{pH}$ of the solution that even a small change in solute concentration caused detectable changes in the color intensity of the solution. Regarding the resolution of the technique, it's worth mentioning that eighteen colors are distinguishable within the full range of solute concentrations used in the experiments. An efficient algorithm was developed in the environment of Scan Pro 5.0 (SPSS) (Theodoropoulou et al., 2003) for the automatic identification of the pore space and solid matrix, recognition of the boundaries of the unit cells (Fig. 2c), and measurement of the intrinsic average colour intensity of each unit cell over various regions of the pore network. With the aid of calibration curves constructed from standard solutions, the colour intensities were converted to solute $(\mathrm{HCl})$ concentrations (Theodoropoulou et al., 2003) .

The glass micromodel was rotated $90^{\circ}$ from the horizontal position so that the gravity acted perpendicular to the main flow direction (Fig. 2b). Single source experiments were performed on each micromodel (Fig. 1), where the dilute $\mathrm{HCl}$ solution was injected at a constant flow rate, $Q_{0}$, through the four inlet ports of the porous medium, and the dense $\mathrm{HCl}$ solution was injected through a central hole (source), at a flow rate $Q_{i}=0.05 Q_{0}$ (Fig. $2 b$ ). Four tubes connected to the outlet ports were kept below the free liquid surface in waste storage tank to avoid, at equilibrium, the vertical component of flow velocity. Snap-shots of the global and local solute dispersion regimes were captured at various time intervals, downstream from the source, and were recorded on the harddisk of the PC for further analysis (Fig. 2b). The transient variation of the spatial distribution of the solute concentration over various regions of the pore network was determined using image analysis technique based on the recorded data.

\section{SOLUTE DISPERSION EQUATIONS}

The steady flow of a liquid of variable density $\rho$ through a porous medium is described by the continuity equation:

$$
\frac{\partial \rho}{\partial t}+\nabla \cdot(\rho \mathbf{u})=0
$$

When the density variation is quite small, the variations of the thermodynamic properties (e.g. density, viscosity, thermal diffusivity, specific heat, and molecular diffusion coefficient) of a fluid are small and the fluid can be regarded as incompressible and can be approximated by Boussinesq equation (Drazin, 2002). The density is assumed independent of pressure and is coupled with the solute concentration $C$ through the linearized approximation (Simmons et al., 2001):

$$
\rho=\rho_{0}\left[1+\beta\left(C-C_{0}\right)\right]
$$

Using tabulated data correlating the $\mathrm{HCl}$ concentration with the density of the corresponding aqueous solution (Perry and Chilton, 1973), it was found that $\beta=0.018 \mathrm{~m}^{3} / \mathrm{kmol}$, whereas $\rho$ varies from $997.7 \mathrm{~kg} / \mathrm{m}^{3}$ to $1004.5 \mathrm{~kg} / \mathrm{m}^{3}$ as the $\mathrm{HCl}$ concentration changes from $C_{0}=0.03 \mathrm{kmol} / \mathrm{m}^{3}$ to $C_{i}=0.41 \mathrm{kmol} / \mathrm{m}^{3}$. According to the Boussinesq approximation, the flow can be regarded as incompressible without any significant loss of accuracy so that Equation (1) is simplified to:

$$
\nabla \cdot \mathbf{u}=0
$$

Under the action of the gravity vector, $\mathbf{g}$, Darcy law is written:

$$
\mathbf{u}=-\frac{\langle k\rangle f(x, y)}{\phi \mu} \nabla(P-\rho \mathbf{g})
$$

where the scalar function $f(x, y)$ represents the spatial variation of the local permeability from its average value $\langle k\rangle$. The solute mass balance is expressed by the well-known advection-dispersion equation (Whitaker, 1999):

$$
\frac{\partial C}{\partial t}+\nabla \cdot(\mathbf{u} C)=\nabla \cdot(\mathbf{D} \cdot \nabla C)
$$

where the components of $\mathbf{D}$ may commonly be expressed as functions of both the flow field and longitudinal $\left(a_{L}\right)$ and transverse $\left(a_{T}\right)$ dispersivities (Zhang et al., 1997; Bruggeman, 1999).

In dimensionless form, Equations (3) and (4) are written:

$$
\begin{gathered}
\frac{\partial u_{\xi}}{\partial \xi}+\frac{\partial u_{\zeta}}{\partial \zeta}=0 \\
u_{\xi}=-\frac{f^{*}(\xi, \zeta)}{\phi} \frac{\partial P^{*}}{\partial \xi} \\
u_{\zeta}=-\frac{f^{*}(\xi, \zeta)}{\phi}\left(\frac{\partial P^{*}}{\partial \zeta}+R a_{1}+R a C^{*}\right)
\end{gathered}
$$

By introducing the dimensionless stream function $\Psi^{*}$ defined by the relationships:

$$
u_{\xi}=\frac{\partial \Psi^{*}}{\partial \zeta} \quad u_{\zeta}=-\frac{\partial \Psi^{*}}{\partial \xi}
$$

and eliminating the pressure between Equations (6) and (7), we get:

$$
\frac{\partial}{\partial \xi}\left(\frac{1}{f^{*}} \frac{\partial \Psi^{*}}{\partial \xi}\right)+\frac{\partial}{\partial \zeta}\left(\frac{1}{f^{*}} \frac{\partial \Psi^{*}}{\partial \zeta}\right)-\frac{R a}{\phi} \frac{\partial C^{*}}{\partial \xi}=0
$$

In dimensionless form, the solute mass balance, Equation (5), is also written:

$$
\begin{aligned}
\frac{\partial C^{*}}{\partial \tau}+u_{\xi} \frac{\partial C^{*}}{\partial \xi}+u_{\zeta} \frac{\partial C^{*}}{\partial \zeta} & =\frac{\partial}{\partial \xi}\left(D_{\xi \xi} \frac{\partial C^{*}}{\partial \xi}+D_{\xi \zeta} \frac{\partial C^{*}}{\partial \zeta}\right) \\
& +\frac{\partial}{\partial \zeta}\left(D_{\xi \zeta} \frac{\partial C^{*}}{\partial \xi}+D_{\zeta \zeta} \frac{\partial C^{*}}{\partial \zeta}\right)
\end{aligned}
$$


The dimensionless variables and parameters involved in the foregoing relations are defined by:

$$
\begin{gathered}
\tau=\frac{t D_{m}}{W^{2}} \\
C^{*}=\frac{C-C_{0}}{C_{i}-C_{0}} \\
\xi=x / W \quad \zeta=y / W \\
u_{j}=\left(\frac{W}{D_{m}}\right) u_{i} \quad i=x, y \quad j=\xi, \zeta \\
D_{k l}=\left(\frac{1}{D_{m}}\right) D_{i j} \quad \begin{array}{l}
i=x, y \quad k=\xi, \zeta \\
j=x, y
\end{array} \quad l=\xi, \zeta \\
R a_{1}=\frac{W\langle k\rangle \rho_{0} g}{\mu D_{m}} \\
R a=\frac{W\langle k\rangle \rho_{0} \beta g\left(C_{i}-C_{0}\right)}{\mu D_{m}} \\
P e=\frac{u_{0} l_{p}}{D_{m}}
\end{gathered}
$$

In addition, the components of the dimensionless dispersion tensor may be given by (Bruggeman, 1999):

$$
\begin{gathered}
D_{\xi \xi}=\frac{D_{e f f}}{D_{m}}+\frac{a_{T}}{W}\left(u_{\xi}^{2}+u_{\zeta}^{2}\right)^{1 / 2}+\frac{\left(a_{L}-a_{T}\right)}{W} \frac{u_{\xi}^{2}}{\left(u_{\xi}^{2}+u_{\zeta}^{2}\right)^{1 / 2}} \\
D_{\zeta \zeta}=\frac{D_{e f f}}{D_{m}}+\frac{a_{T}}{W}\left(u_{\xi}^{2}+u_{\zeta}^{2}\right)^{1 / 2}+\frac{\left(a_{L}-a_{T}\right)}{W} \frac{u_{\zeta}^{2}}{\left(u_{\xi}^{2}+u_{\zeta}^{2}\right)^{1 / 2}} \\
D_{\xi \zeta}=\frac{\left(a_{L}-a_{T}\right)}{W} \frac{u_{\xi} u_{\zeta}}{\left(u_{\xi}^{2}+u_{\zeta}^{2}\right)^{1 / 2}}
\end{gathered}
$$

From a macroscopic point of view, the parameters that control the solute dispersion process are the Rayleigh number, $R a$, the Peclet number, $P e$, and the dispersivities $a_{L}$ and $a_{T}$. The Rayleigh number, $R a$, expresses the ratio of the buoyancy-driven solute transport to the diffusive solute transport and refers to a characteristic length scale of the porous medium. For sufficiently high $R a$ numbers greater than some critical value gravity-based instability will occur in the form of waves in the boundary layer that develop into fingers or plumes. This critical $R a$ value defines the transition between dispersive/diffusive solute transport (at lower $R a$ values) and convective transport by density-driven fingers (at higher $R a$ values) (Simmons et al., 2001). For a planar 2-D system, like the glass micro-models used here, the values of $\langle k\rangle$ and $\phi$ depend on the selected reference length along the third direction. The usage of a modified Rayleigh number defined by:

$$
R a_{m}=\frac{R a}{\phi}
$$

is preferable.

In the present work, no systematic attempt is done to examine experimentally the effect of $R a_{m}$ on the solute dispersion regime. Neither the properties of the model porous medium $(\langle k\rangle, W, \phi)$ nor the properties of the fluid system $\left(\rho, \beta, C_{i}-C_{0}, D_{m}\right)$ could change in a wide range of parameter values. This is due to inherent limitations of the glass-etching technique (the depth of etched pores ranges from 100 to $200 \mu \mathrm{m}$ ), and restrictions to the solute type (acid) and concentrations used (above a relatively small $\mathrm{HCl}$ concentration, no color intensity changes are visible).

\section{TIME SERIES ANALYSIS}

An acceptable definition of a chaotic concentration field can be related to the value of the dimension of the chaotic attractor. If the dimension is much greater than unity then the concentration field is chaotic as defined by Guckenheimer and Holmes (1986). In order to determine the dimension of the attractor, state-space analysis of the experimental time series is required (Johnson et al., 2000). All methods of the nonlinear time-series analysis are based on the construction of an attractor of the dynamic evolution of the system in state-space. The method of reconstruction in state-space (embedding) is theoretically (Takens, 1981) based on the fact that all information needed to define the state of the system exists in a time series of one single measured parameter, such as the fluctuating solute concentration measured locally in the pore network. The most common methods to characterize the attractor are the evaluation of the correlation dimension and the Kolmogorov entropy (Grassberger and Procaccia, 1984) and/or determination of the Lyapunov exponents (Abarbanel, 1990). Because of the small number of measurements that were acquired in each experiment, no attempt was done to determine the attractor from the solute concentration time series. Instead the linear methods of time domain and frequency domain analysis were used.

\subsection{Time Domain Analysis}

All analyses are made on time-series with data points $z(n)$, with $n=1,2,3, \ldots N$, measured at equidistant time intervals 
$\Delta \tau$, where $N$ is the total number of measurements, and $z$ is the variable signal. The amplitude of the signal is expressed by the standard deviation:

$$
\sigma=\sqrt{\frac{1}{\mathrm{~N}-1} \sum_{n=1}^{N}(z(n)-\bar{z})^{2}}
$$$$
\text { with the average: } \quad\langle z\rangle=\frac{1}{N} \sum_{n=1}^{N} z(n)
$$

The higher order statistical moments are expressed in a dimensionless form as the skewness (normalized 3rd-order moment):

$$
S=\frac{1}{N \sigma^{3}} \sum_{n=1}^{N}(z(n)-\bar{z})^{3}
$$

and the flatness (normalized 4th-order statistical moment):

$$
F=\frac{1}{N \sigma^{4}} \sum_{n=1}^{N}(z(n)-\bar{z})^{4}
$$

The skewness indicates the lack of symmetry in the probability distribution and is zero for a normal distribution of noise. The flatness is a measure of the sharpness of the distribution, is 3 for normal Gaussian distribution, and can be regarded as the ratio of the time spent under quiescent conditions to the time spent under active conditions. The correlation between two points separated by a time lag $\tau_{k}=k \Delta \tau$ is expressed by the autocorrelation function, which in normalized form, is given by:

$$
c\left(\tau_{k}\right)=\frac{\sum_{n=1}^{N-\tau_{k}}\left[\left(z_{n+\tau_{k}}-\langle z\rangle\right)\left(z_{n}-\langle z\rangle\right)\right]}{\sum_{n=1}^{N}\left(z_{n}-\langle z\rangle\right)^{2}}
$$

The autocorrelation for a periodic signal is also periodic and for deterministic chaotic systems the autocorrelation function decays exponentially with increasing lag (Dimitrova and Vitanov, 2001). When the autocorrelation function falls abruptly to zero, that indicates the lack of a deterministic component from the data; a slow fall to zero is a sign of stochastic or deterministic behavior; when the data slowly drops to zero and shows periodic behavior then the data is highly correlated and is either periodic or chaotic in nature.

\subsection{Frequency Domain Analysis}

The power spectral density (power spectrum) of a signal is defined by:

$$
p(f)=|Z(f)|^{2}+|Z(-f)|^{2} \quad 0 \leq f \leq \infty .
$$

where $Z(f)$ is the Fourier transform of the investigated signal $z(\tau)$. The power spectrum of a discrete signal is identical to the real part of the FFT, and give us information on how much power is contained in each frequency. Thus, we can visualize the dominant frequencies associated with the system dynamics and we can investigate their shifting when the system parameters change. Random and chaotic datasets fail to demonstrate a dominant frequency. Periodic or quasiperiodic datasets show a dominant or several frequencies. By definition, a periodic signal is governed by a single fundamental frequency or its simple multiples in the power spectrum. Quasi-periodic signals are governed by two fundamental frequencies or its multiples, whereas chaotic signals comprise three or more fundamental frequencies (Basu and Islam, 1996; Dimitrova and Vitanov, 2001). The interpretation of power spectra is subjective. What is regarded to be a peak for the determination of a dominant frequency may differ between observers. The shape of a spectrum depends on the number of samples, the sampling frequency, and the number of the spectra averaged (Johnson et al., 2000).

Both deterministic/chaotic and stochastic systems have a strong decay in autocorrelation function with time lag, and broad banded power spectra (Baker and Gollub, 1996). The link between the shape (fall-off with frequency, $f$ ) of the power spectrum and the type of system (chaotic/deterministic or stochastic) on measured time series may be used to characterize the system. Specifically, the fall-off of power spectrum at high frequencies follows a power law for stochastic and multifractal systems and an exponential law for systems exhibiting deterministic chaos (Johnson et al., 2000).

\section{RESULTS AND DISCUSSION}

\subsection{Estimation of Longitudinal and Transverse Dispersivities}

To determine the effective diffusion coefficient, $D_{\text {eff }}$, the following relationship can be used (Sahimi, 1995):

$$
\frac{D_{e f f}}{D_{m}}=\frac{1}{F_{f} \phi}
$$

where the formation factor $F_{f}$ can be either measured or calculated (Tsakiroglou, 2002). There is an ambiguity concerning the definition of the porosity of a planar pore network. With reference to a unit cell of four interconnected capillaries in a square lattice (Fig. 2c), the areal porosity $\phi_{a}$ is given by:

$$
\phi_{a}=\frac{\left\langle w_{p}\right\rangle\left(2 l_{p}-\left\langle w_{p}\right\rangle\right)}{l_{p}{ }^{2}}
$$

The volumetric porosity $\phi$ is defined as the ratio of the pore volume to a reference total volume with height equal to $l_{p}$. Finally, we get:

$$
\phi=\phi_{\alpha} \frac{\pi\left\langle d_{p}\right\rangle}{4 l_{p}}
$$


For the model M-1 it was found that $D_{\text {eff }} / D_{m}=0.56$ $\left(F_{f \exp }=44.42, \phi=0.04\right)$ and this value was set as the lower limit to the estimated $D_{\xi \xi}$ and $D_{\zeta \zeta}$ values.

In a horizontal experiment without the action of gravity, the steady-state solute dispersion regime depends on $P e$ and remains unchanged in time (Theodoropoulou et al., 2003). If the $\xi$-component of the flow velocity is constant throughout the pore network, $u_{\xi}=\left(W / D_{m}\right) u_{0}$, and the $\zeta$-component is negligible, $u_{\zeta}=0$, then:

$$
\begin{aligned}
& D_{\xi \zeta}=0 \\
& D_{\xi \xi}=\frac{D_{e f f}}{D_{m}}+\frac{a_{L} u_{0}}{D_{m}} \\
& D_{\zeta \zeta}=\frac{D_{e f f}}{D_{m}}+\frac{a_{T} u_{0}}{D_{m}}
\end{aligned}
$$

With this simplification it is easy to solve the advectiondispersion equation analytically (Theodoropoulou et al., 2003). The longitudinal and transverse dispersion coefficients were estimated by fitting the measured transient and steady-state solute concentration from experiments performed on model M-1 to the analytical solution of the 2D advection-dispersion equation (Theodoropoulou et al., 2003). However, both $D_{\xi \xi}$ and $D_{\zeta \zeta}$ values were overestimated (see Figure 17 in Theodoropoulou et al., 2003) because the cross-section of the solute source was regarded as rectangular with dimensions equal to $b$ and $l_{p}$. In reality, the solute is injected through a disk (Fig. 2b) with dimensions equal to $P_{s}=\pi b$ and $l_{p}$ (note that the pore length was used as a reference length for defining the porosity and permeability of the planar network). The values of $D_{\xi \xi}$ and $D_{\zeta \zeta}$ were reestimated using the solute fluxes based on a cross-sectional area equal to $\pi b l_{p}$, and fitting the analytic equation to the experimentally measured solute concentration profiles using Bayesian estimator of the Gregplus solver of Athena (Stewart and Associates, Inc.) software package (Theodoropoulou et al., 2003). Two estimation procedures were adopted:

- the values of $D_{\xi \xi}$ and $D_{\zeta \zeta}$ were estimated by fitting simultaneously the transient and steady-state solute concentration profiles to the (transient) analytical solution of the advection-dispersion equation;

- the following asymptotic steady-state solution $\left(\partial C^{*} / \partial \tau=0\right)$ of the advection-dispersion equation is obtained:

$$
\begin{aligned}
C^{*} & =\frac{1}{\left(C_{i}-C_{0}\right)} \\
& \left\{\frac{1}{W} \frac{I_{0}}{u_{0}}+\sum_{n=1}^{\infty} \frac{I_{n}}{r_{n}} \cos \left(\lambda_{n} y\right) \exp \left[\left(\frac{u_{0}}{2 D_{\xi \xi} D_{m}}-p_{n}\right) \xi W\right]\right\}
\end{aligned}
$$

where the parameters involved in Equation (34) are given by:

$$
\begin{gathered}
\lambda_{n}=\frac{n \pi}{W} \\
I_{0}=b \frac{F_{m}}{\phi} \\
I_{n}=\frac{2}{\pi n} \frac{F_{m}}{\phi}\left\{\sin \left[\lambda_{n}\left(d_{s}+b\right)\right]-\sin \left(\lambda_{n} d_{s}\right)\right\} \\
p_{n}=\sqrt{\left(\frac{D_{\zeta \zeta}}{D_{\xi \xi}}\right) \lambda_{n}{ }^{2}+\left(\frac{u_{0}}{2 D_{\xi \xi} D_{m}}\right)^{2}} \\
r_{n}=\left[\frac{u_{0}}{2}+\sqrt{D_{\xi \xi} D_{\zeta \zeta} D_{m}{ }^{2} \lambda_{n}{ }^{2}+\frac{u_{0}{ }^{2}}{4}}\right] \\
\frac{F_{m}}{\phi}=\frac{Q_{s}\left(C_{i}-C_{0}\right)}{\pi b l_{p}}=\left(C_{i}-C_{0}\right)\left[u_{0} C^{*}-\left(\frac{D_{\xi \xi} D_{m}}{W}\right) \frac{\partial C^{*}}{\partial \xi}\right]_{\xi=0}
\end{gathered}
$$

Parametric analysis has shown that the value of $D_{\xi \xi}$ affects weakly on the calculated solute concentration profile. The $D_{\zeta \zeta}$ was first estimated by fitting steady-state datasets to Equation (34), and afterwards the $D_{\zeta \zeta}$ was estimated by fitting the transient data to the corresponding (transient) analytic solution (Table 2). The fluctuation of $D_{\xi \xi}$ and $D_{\zeta \zeta}$ values near very low $P e$ numbers (Table 2 ) may be attributed to the deviation of the real boundary condition at the exit (finite porous medium, four outlet ports) from those assumptions made to derive them analytically (infinite porous medium, $\partial C^{*} / \partial \xi=0$ as $\xi \rightarrow \infty$ ). The finite length of the porous medium does not influence the solute concentration profiles at the very early times, but affects drastically the flow field near the exit, and subsequently the long-term transient and steady-state solute concentration profiles.

According to Equations (14), (19) and (20) the dispersivities $a_{L}$ and $a_{T}$ are given by:

$$
\begin{aligned}
& \frac{a_{L}}{l_{p}}=\left(D_{\xi \xi}-\frac{D_{e f f}}{D_{m}}\right) \frac{1}{P e} \\
& \frac{a_{T}}{l_{p}}=\left(D_{\zeta \zeta}-\frac{D_{e f f}}{D_{m}}\right) \frac{1}{P e}
\end{aligned}
$$

In order to determine reasonable dispersivities, the values of $D_{\xi \xi}, D_{\zeta \zeta}$ values estimated using the 1 st estimation approach for $P e=0.5,2.5$, whereas the 2nd approach was used to estimate $D_{\xi \xi}$ and $D_{\zeta \zeta}$ for $P e=1.0,5.0,10.0$ (Table 2). Evidently, the estimated $a_{L}$ and $a_{T}$ values are subjected to higher uncertainty that is governed by $P e$, the quantity and quality of dataset, the procedure of parameter estimation, as well as the choice of the analytical model $C^{*}=C^{*}(\xi, \zeta, \tau)$. The dimensionless values of $P e$ and $R a_{m}$ corresponding to the experimental conditions are summarized in Table 3. 
TABLE 2

Dispersion coefficients estimated from experimental datasets of model M-1

\begin{tabular}{l|c|c|c|c|c|c}
\hline & \multicolumn{2}{|c|}{1 st approach } & \multicolumn{2}{c|}{ 2nd approach } & \multirow{2}{*}{$a_{L}(\mathrm{~m})$} & \multirow{2}{*}{$a_{T}(\mathrm{~m})$} \\
\hline$P e$ & $D_{\xi \xi}$ & $D_{\zeta \zeta}$ & $D_{\xi \xi}$ & $D_{\zeta \zeta}$ & & \\
\hline 0.5 & $1.45 \pm 0.017$ & $0.745 \pm 0.062$ & - & $3.02 \pm 0.19$ & 0.00243 & 0.00050 \\
1.0 & $4.86 \pm 1.8$ & 0.56 & $3.23 \pm 1.77$ & $0.643 \pm 0.068$ & 0.00364 & 0.00011 \\
2.5 & $1.73 \pm 0.69$ & $0.622 \pm 0.111$ & - & $1.684 \pm 0.104$ & 0.00064 & 0.000034 \\
5.0 & $11.1 \pm 13.3$ & 0.56 & $11.85 \pm 14.7$ & $0.67 \pm 0.17$ & 0.00310 & 0.00003 \\
10.0 & $28.8 \pm 8.15$ & 0.56 & $28.7 \pm 41.1$ & $0.92 \pm 0.1$ & 0.00385 & 0.00005 \\
\hline
\end{tabular}

TABLE 3

Dimensionless parameter values used in experiments

\begin{tabular}{l|c|c|c}
\hline Parameter & Model M-1 & Model S-1 & Model D-1 \\
\hline$P e$ & $0.5,1.0,2.5,5.0,10.0$ & $3.7,5.6$ & $3.3,4.9$ \\
$R a_{m}$ & 1060 & 2085 & 1650 \\
\hline
\end{tabular}

\subsection{Gravity Effects on Solute Dispersion Regimes}

In the present work, we used conventional linear methods of analysis, and conclusions pertinent to the system behavior were drawn from the properties of the autocorrelation function and power spectrum of the temporal evolution of the solute concentration.

Successive snap-shots of the solute dispersion in the model pore network M-1 at various times and for several $P e$ numbers are shown in Figures 3a-c. The solute dispersion plume exhibits a fluctuating behavior reflected in the lobeshaped instabilities. The transient evolution of the lobes along the plume have the characteristics of a periodic or chaotic behavior (Fig. 3). The multiple of steady-state solute concentration profiles are caused by the downward flow of the heavier solution injected at the source and the upward flow of the lighter solution injected at the inlet ports. The longitudinal dispersivity remains almost unchanged, exhibiting a local minimum at $P e=2.5$, whereas the transverse dispersivity monotonically decreases as the $P e$ number increases (Table 2). The lobe-shaped instabilities become distinguishable at $P e=2.5$ (Fig. $3 b$ ) and this is in agreement with earlier macroscopic numerical studies predicting that the buoyancy-driven instabilities are favored by small values of the dispersivities (Liu and Dane, 1997). At higher $P e$ numbers, the longitudinal dispersion coefficient increases while the transverse dispersion coefficient remains almost constant (Table 2). In this manner, the thickness of the dispersion zone and buoyancy-driven lobes is reduced, and the solute dispersion regime tends asymptotically to be stabilized, although the multiple of steady-states is still observed (Fig. 3c).

A small region in the pore network $\mathrm{M}-1$, indicated in square box in Figure $3 \mathrm{~b}$ is blown up in Figure 4 to show how the solute concentration varies with the time without converging to any stable solute concentration distribution. The solute dispersion regime (Fig. 4a) is converted to equiconcentration contours (Fig. 4b) that illustrate the fluctuations of the local solute concentration at smaller scales. Evidently, the experimental technique enables us to quantify local fluctuations of the solute concentration at different scales ranging from a unit cell of four interconnecting pores (Fig. $4 a$ ) to the pore network scale (Fig. 3b). The average solute concentration over a small region of the model M-1 (Figs. 3b, 4a) was plotted as a function of time and the mean period between two neighboring maxima or minima were helpful to identify eventual periodic fluctuations (Fig. 5a). An almost constant mean period of fluctuations was found (Fig. 5a). This is indicative of the periodic nature of the solute dispersion regime. The autocorrelation function tends slowly to zero (indicative of stochastic or deterministic processes) with fluctuations that indicate some form of periodic behavior (Fig. 5b). The amplitude of the fluctuations is quite large compared to their average $\left(\sigma /\left\langle C^{*}\right\rangle \cong 0.4\right)$, they are asymmetric $(S=0.51)$ and flatter than the Gaussian noise $(F=2.47)$. No dominant frequency is evident in the power spectrum (Fig. $5 c$ ) and hence we are unable to draw any clear conclusion about the periodic or chaotic nature of the fluctuations from frequency domain analysis. Actually, the number of measurements is so small, or equivalently the frequency range is so narrow that the identification of dominant frequencies is impossible.

The steady-state solute dispersion regimes in the single pore network S-1 are shown in Figure 6. At low $P e$ values, the mixing zone is very wide reaching the lower boundary of the pore network (Fig. 6a) so that it is difficult to distinguish clearly any individual lobes. The fluctuations are characterized by a broad amplitude $\left(\sigma /\left\langle C^{*}\right\rangle=0.67\right)$, and the lack of periodicity (Fig. $7 a$ ). Likewise, no periodicity is evident in the oscillations of the autocorrelation function (Fig. 7b) whereas a broad band FFT power spectrum with a negative fall-off slope appears in the frequency domain (Fig. 7c). Such a dispersion regime can be considered as chaotic although the aforementioned characteristics of the solute concentration time series are simply indicative of such 

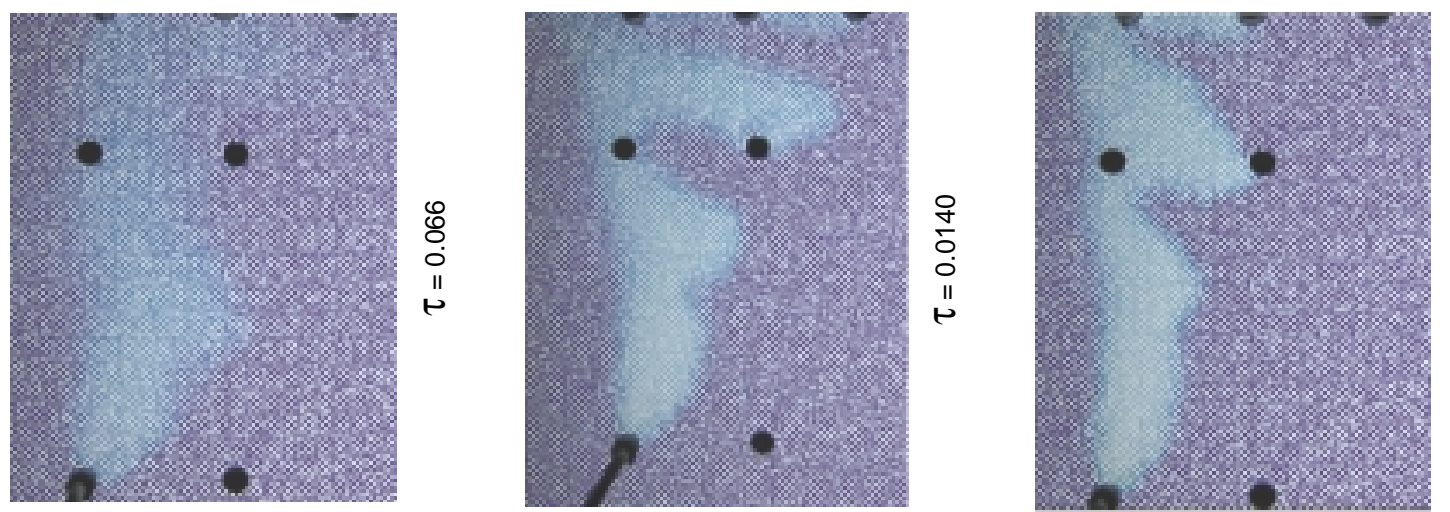

f
0
0
11
•
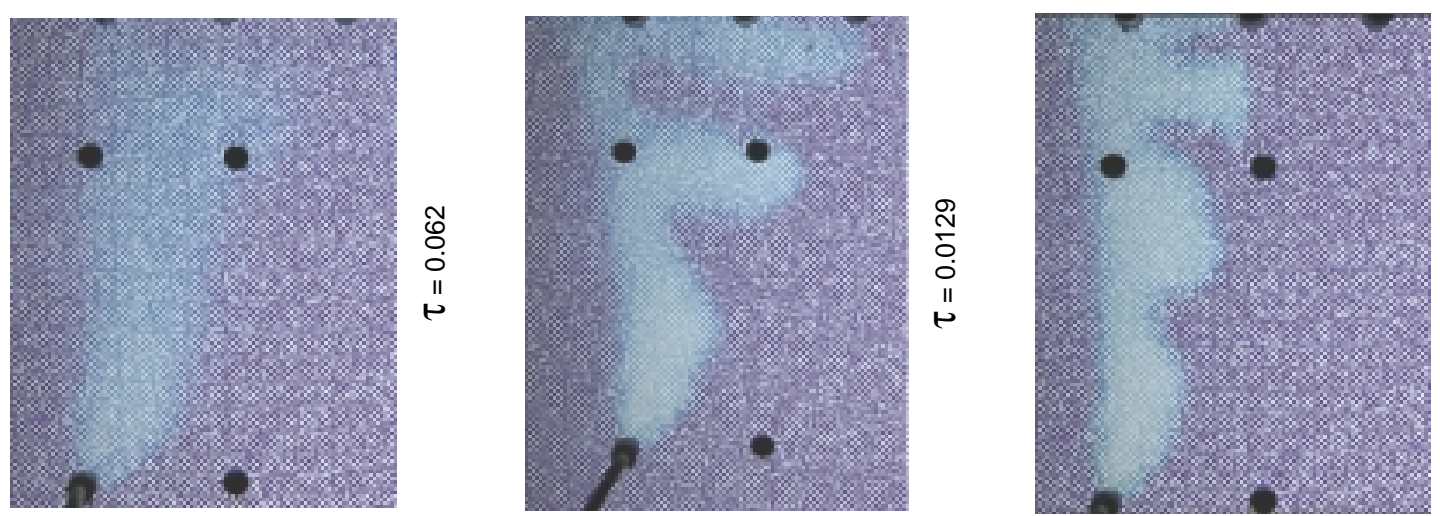

$\overline{0}$
0
0
11
0
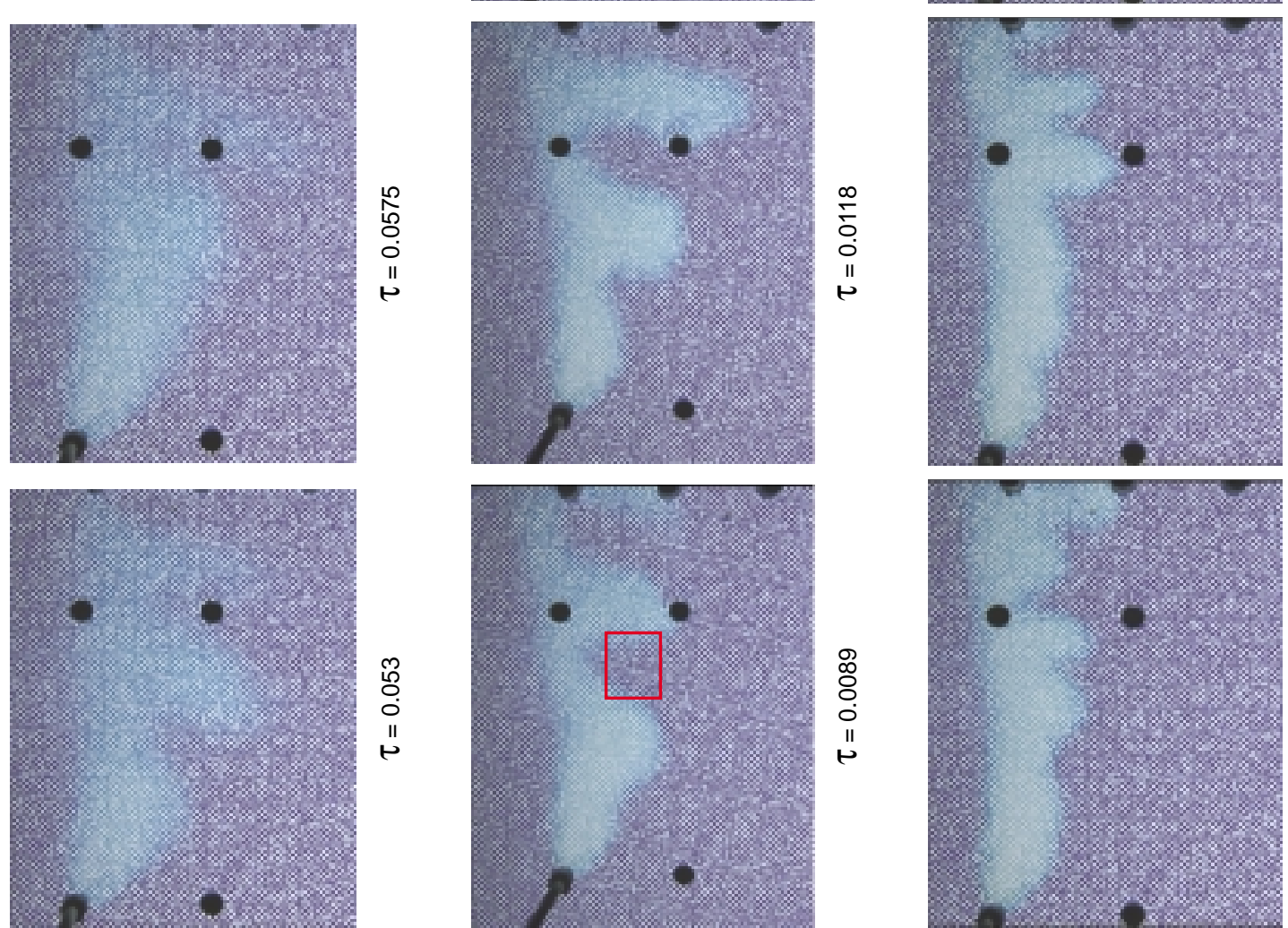

ส

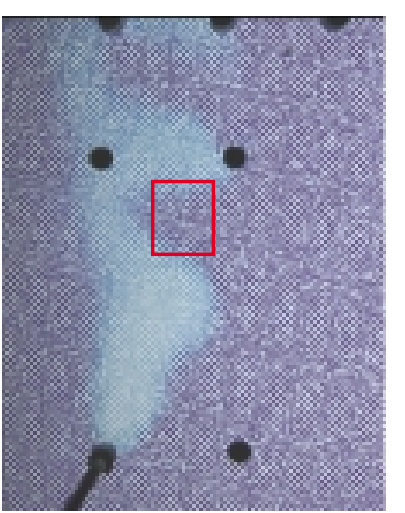

อ

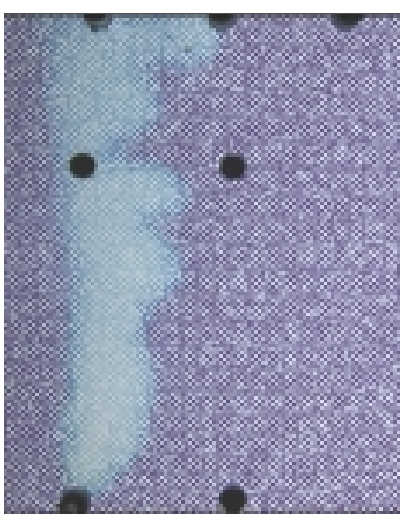

ธิ

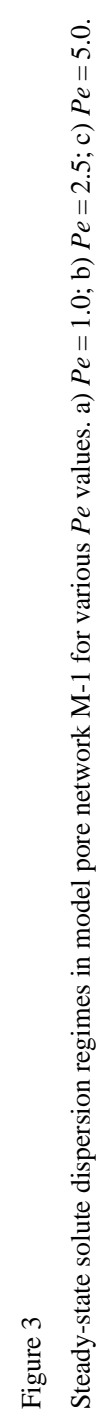



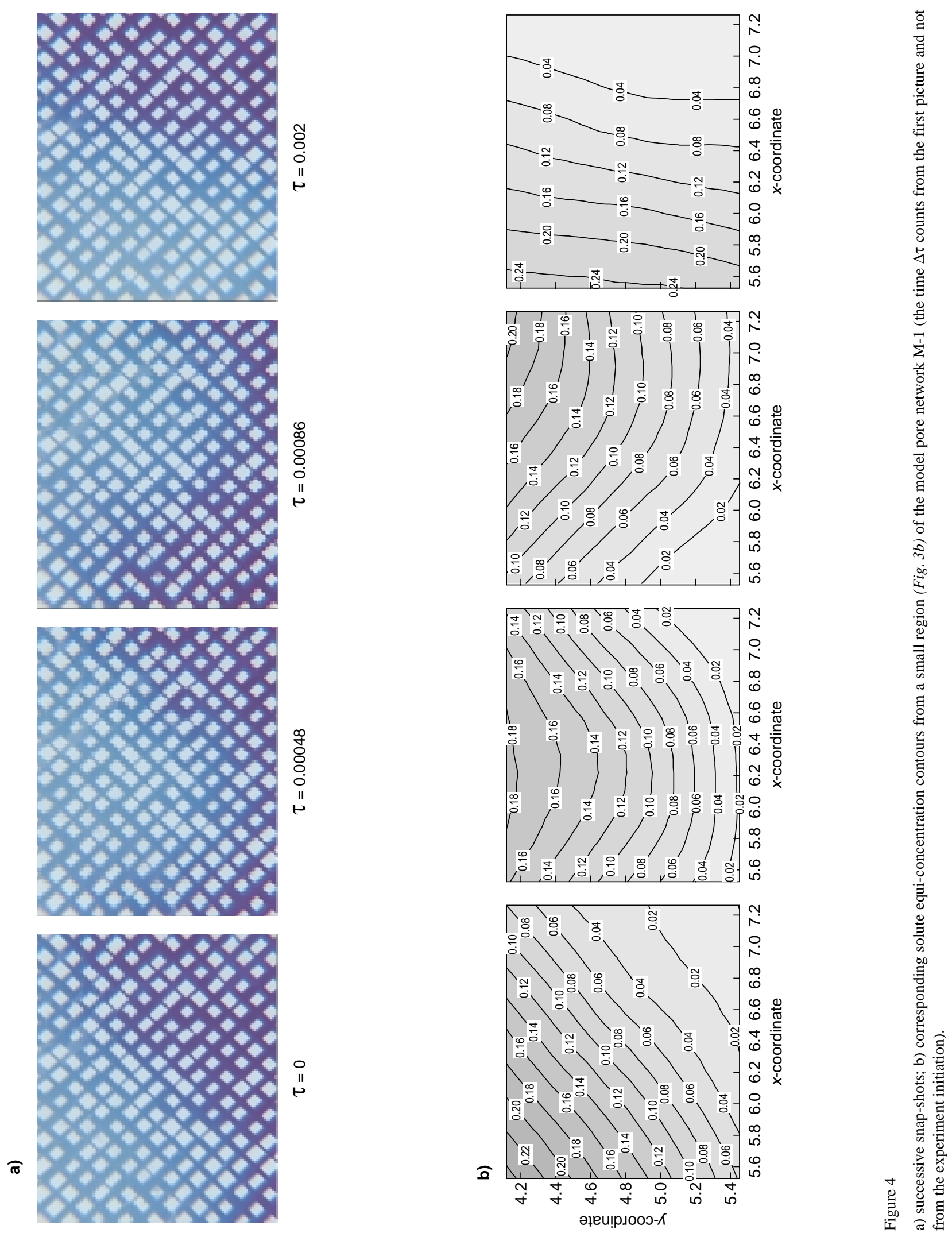

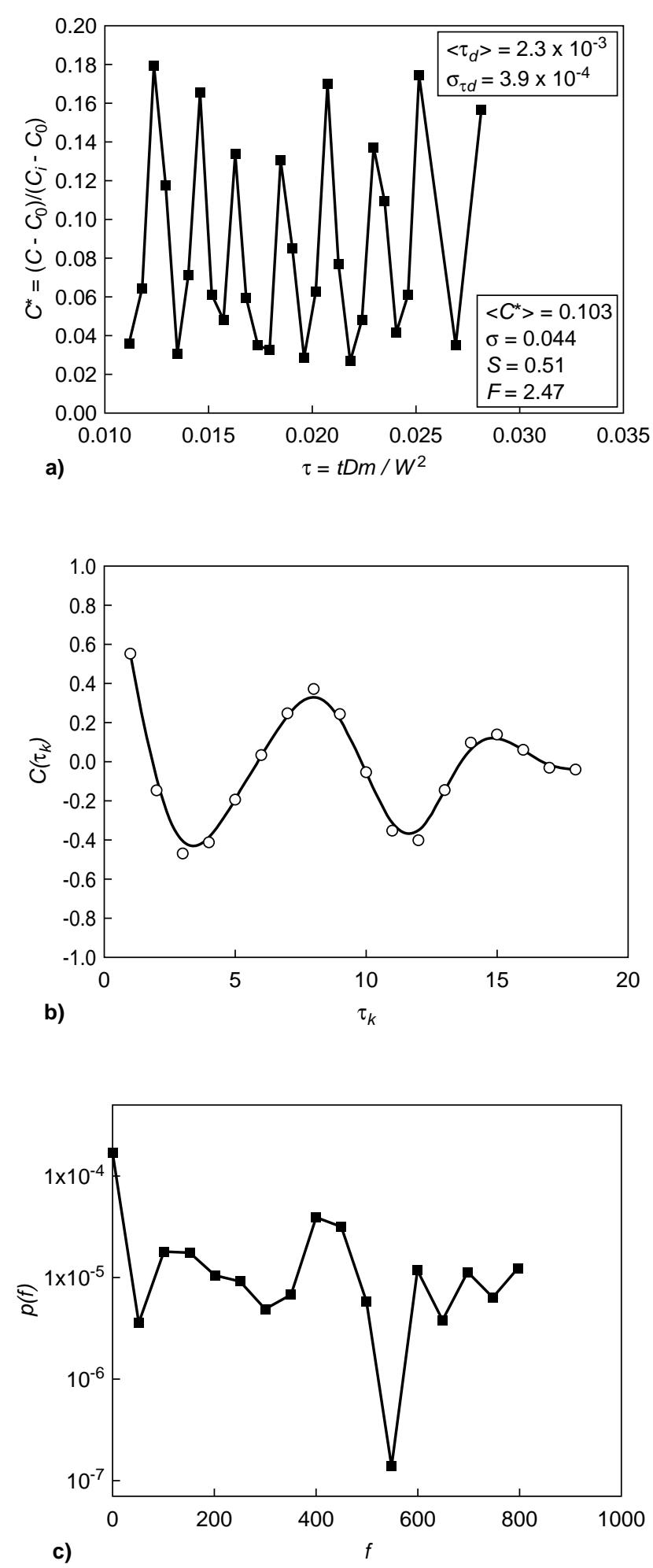

Figure 5

a) temporal evolution; b) autocorrelation function; c) FFT power spectrum of the average solute concentration over a small region (Fig. $3 b$ ) of the model pore network M-1 for $P e=2.5$. a behavior (Johnson et al., 2000). At higher Pe values, unstable lobes of finite thickness smaller than the width of the network are created (Fig. $6 b$ ). The amplitude of the concentration fluctuations is almost constant with two characteristic periods to be identified in the time domain (Fig. 8a). The fluctuations are slightly asymmetric $(S=0.21)$ and much flatter than a Gaussian noise $(F=1.53)$ whereas some form of periodicity may appear in the oscillations of the autocorrelation function (Fig. 8b). The broad band FFT power spectrum that decays exponentially at high frequencies (Fig. 8c) is typical of chaotic motions. The dispersion regime (Fig. 6b) may be regarded as a quasiperiodic or chaotic one, but additional information concerning the behavior of the solute concentration series at long times is required before such a classification is done. In other words, we are unable to classify clearly the solute dispersion regime because of the small number of experimental measurements that are available to time domain and frequency domain analyses.

The steady-state solute dispersion regimes in the dual pore network D-1 are shown in Figure 9. At relatively low $P e$ values, the length of the lobes is quite large and the solute concentration is characterized by slightly asymmetric $(S=0.37)$ and very flat $(F=1.45)$ fluctuations of high amplitude $\left(\sigma /\left\langle C^{*}\right\rangle=0.46\right)$ (Fig. 9a). The lack of a constant period in the time series (Fig. 10a), the periodicity observed on the oscillations of the autocorrelation function before their decay to zero (Fig. 10b) and the three fundamental frequencies identified on the power spectrum (Fig. 10c) are indicative of a chaotic dispersion regime. At higher $P e$ values, the dispersion regime is dominated by unstable lobes of smaller length, and progressively it tends to be stabilized (Fig. 9b). Three different periodic fluctuations of the concentration are evident in the time domain for $P e=4.9$ (Fig. 11a). The autocorrelation function decreases slowly toward zero by exhibiting some oscillations (Fig. 11b), whereas a broad band power spectrum without any dominant frequency is observed (Fig. 11c). The foregoing characteristics may be regarded indicative of a chaotic dispersion regime with some uncertainty associated with the narrow time/frequency range of the experimental measurements.

By comparing the dispersion regimes observed in model S-1 with those observed in model D-1 some information can be deduced with respect to the role of pore scale heterogeneities on buoyancy driven instabilities. It seems that the existence of the network of thin and short pores has a stabilizing effect on the dispersion regime by reducing the thickness of the mixing zone at the corresponding Pe value (Figs. $6 a$ and $9 a$ ). On the other hand, the high degree of pore-scale heterogeneities embedded into the dual pore network D-1 favors the onset of chaotic regimes even at relatively high $P e$ values, where the length of the unstable lobes is finite and the lateral boundaries don't influence the evolution of the dispersion within the pore network (Fig. 9b). 

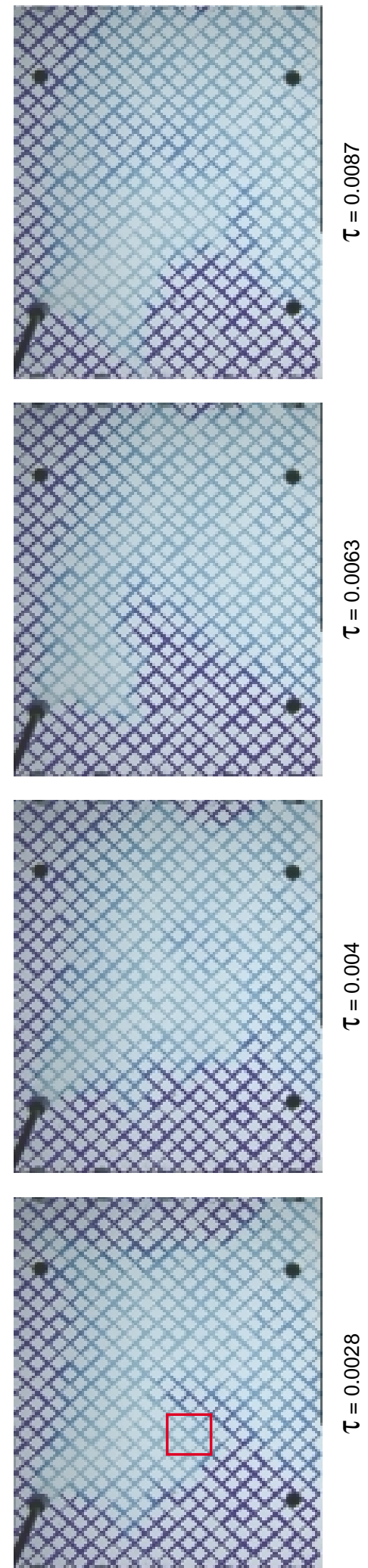

ส
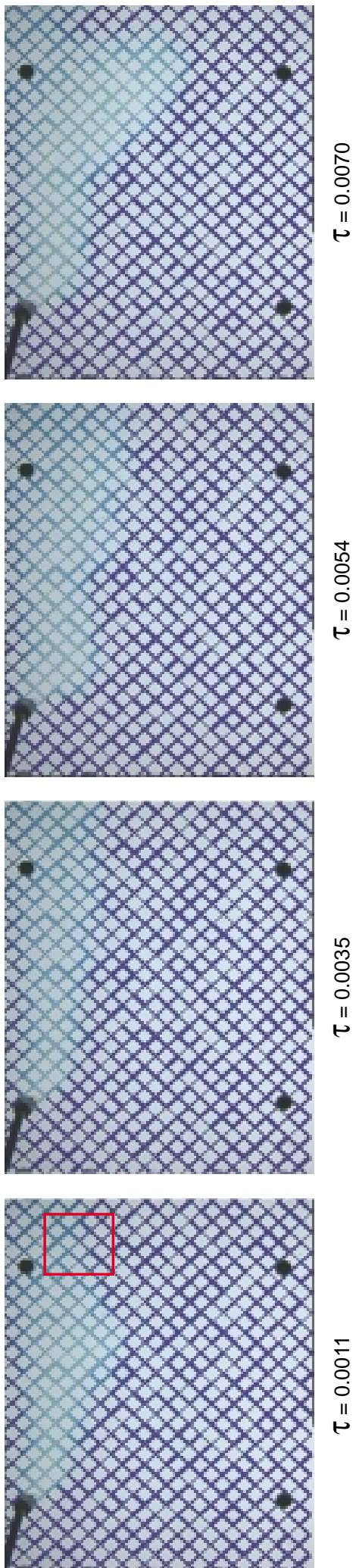

อ

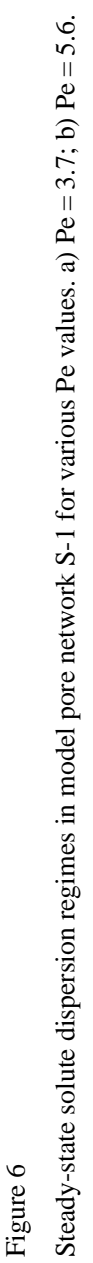



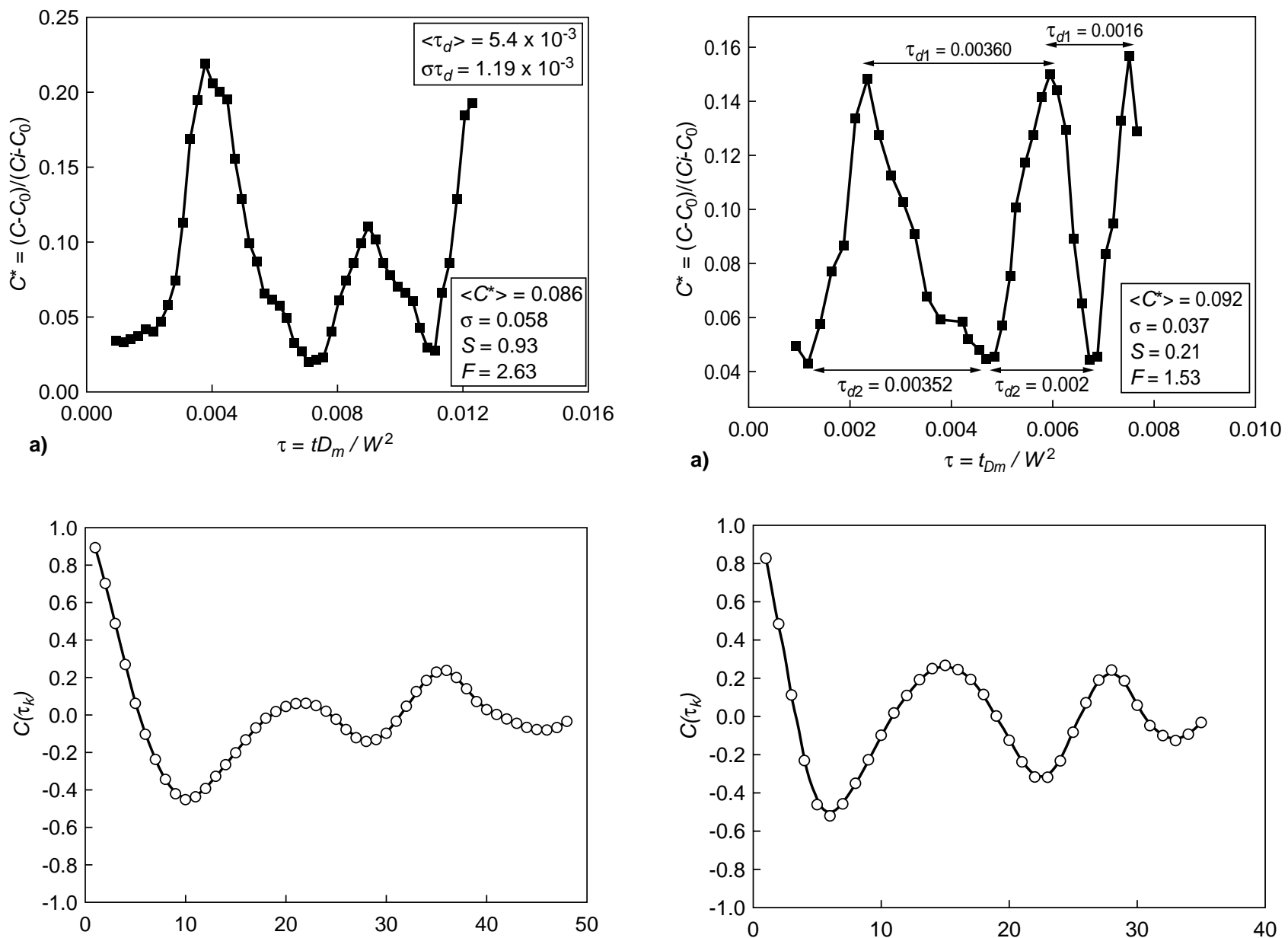

b)

$\tau_{k}$
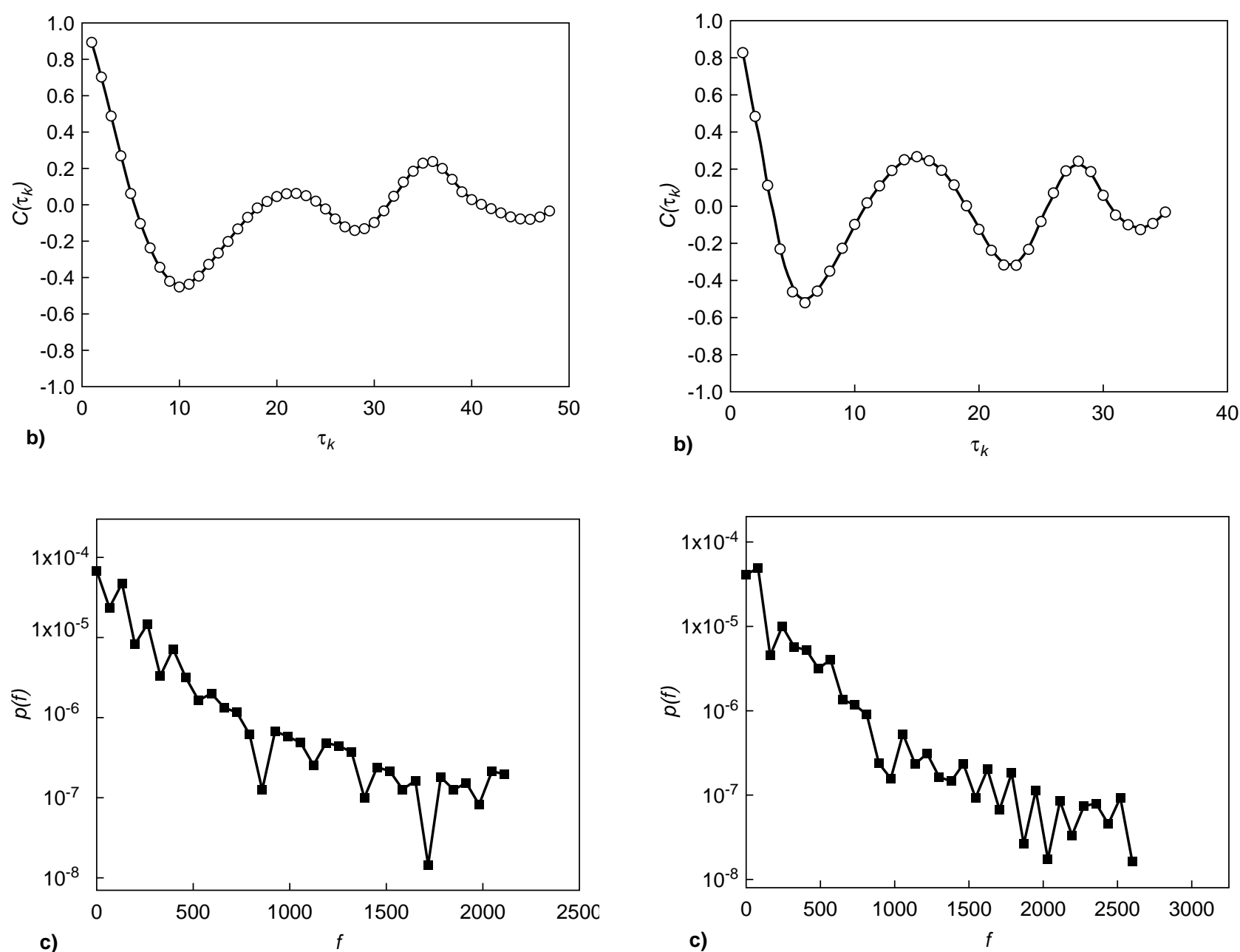

Figure 7

a) temporal evolution; b) autocorrelation function; c) FFT power spectrum of the average solute concentration over a small region (Fig. 6a) of the model pore network S-1 for $P e=3.7$.

Figure 8

a) temporal evolution; b) autocorrelation function; c) FFT power spectrum of the average solute concentration over a small region (Fig. 6b) of the model pore network S-1 for $\mathrm{Pe}=5.6$. 

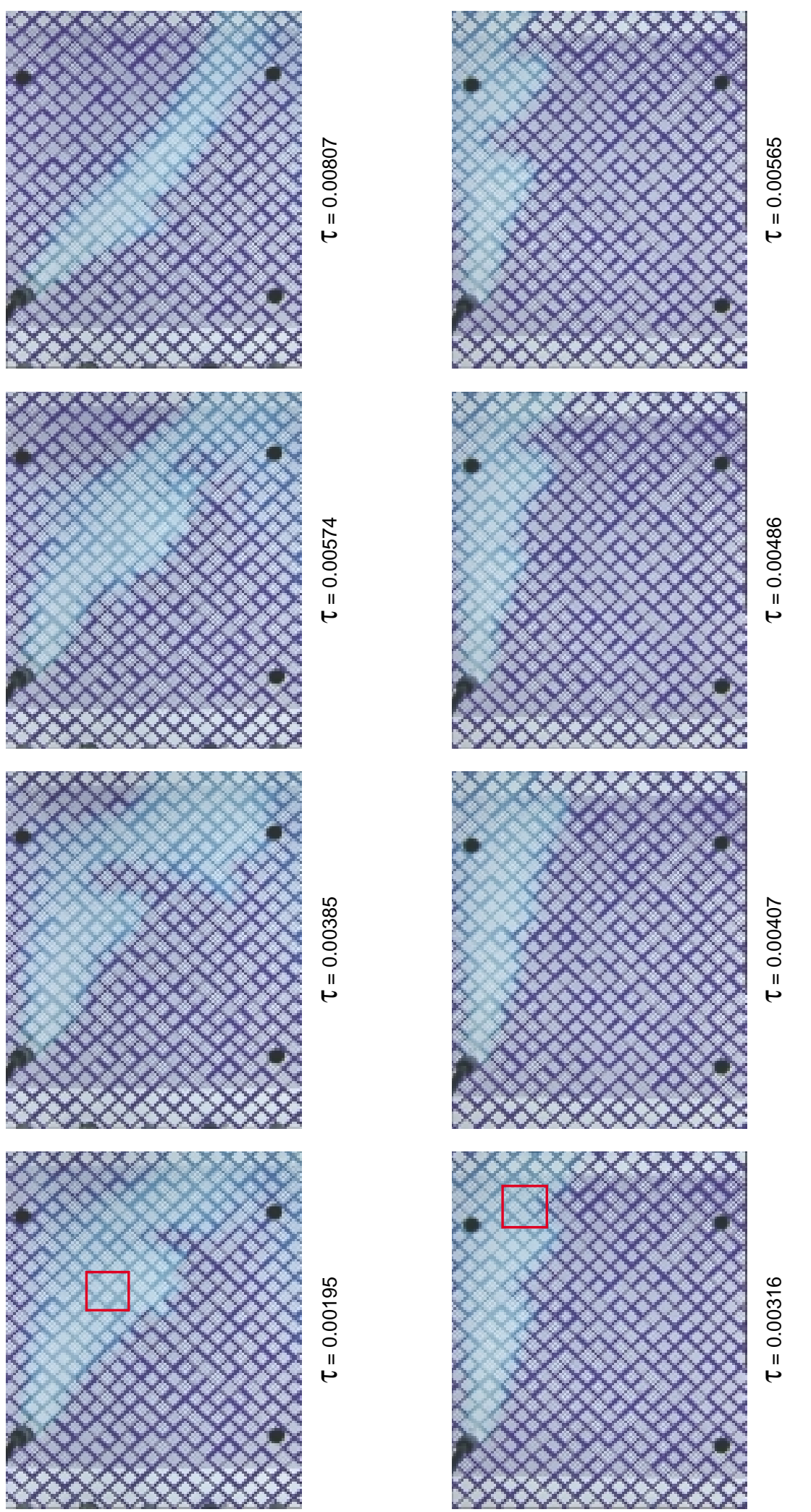

$\widehat{\sigma}$

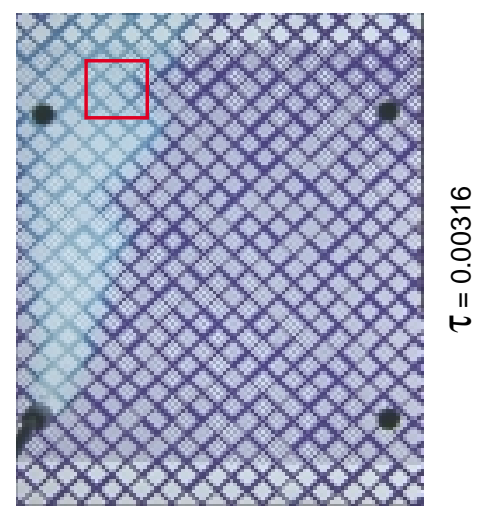

อ

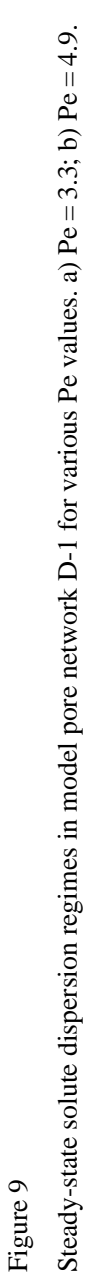



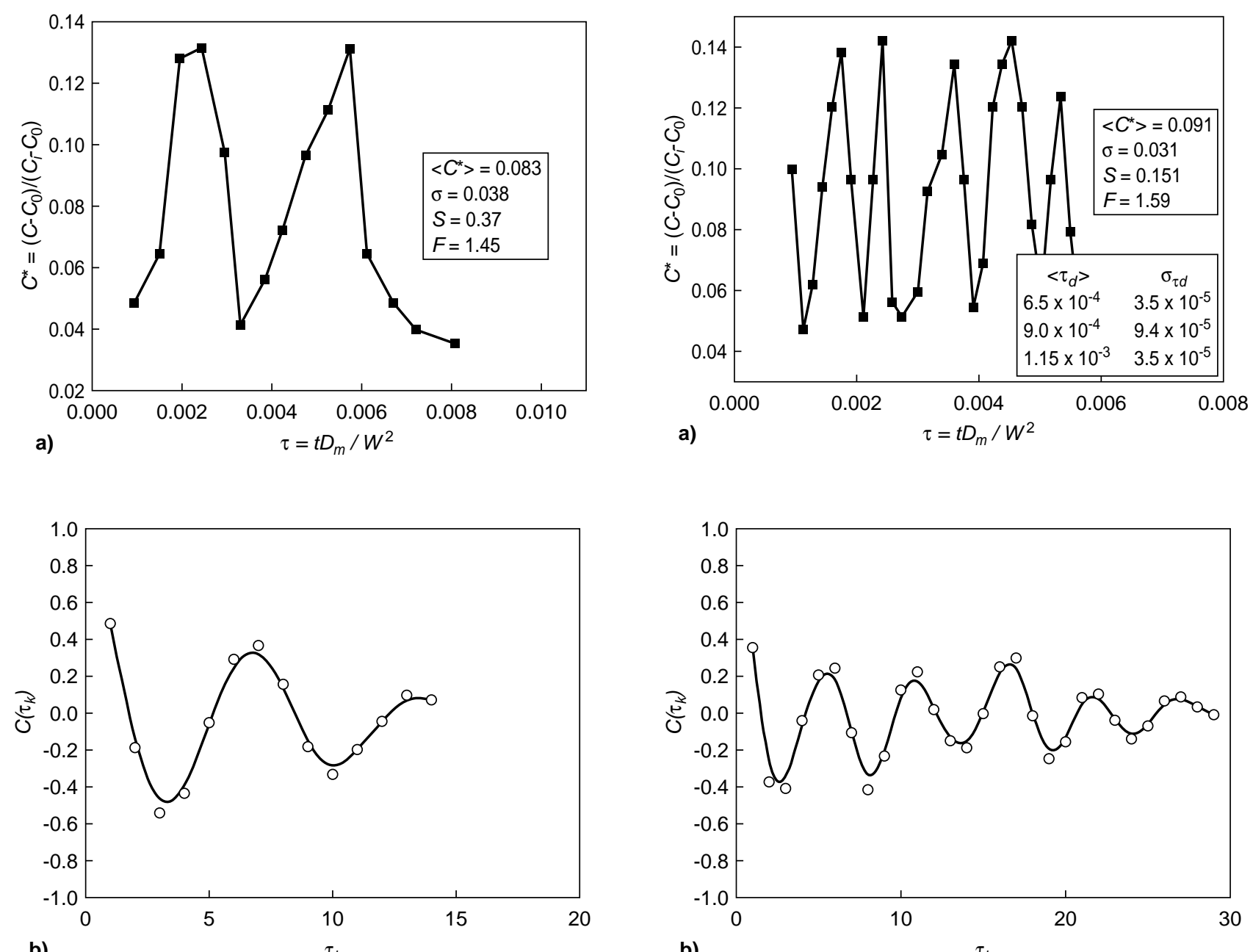

b)

b)

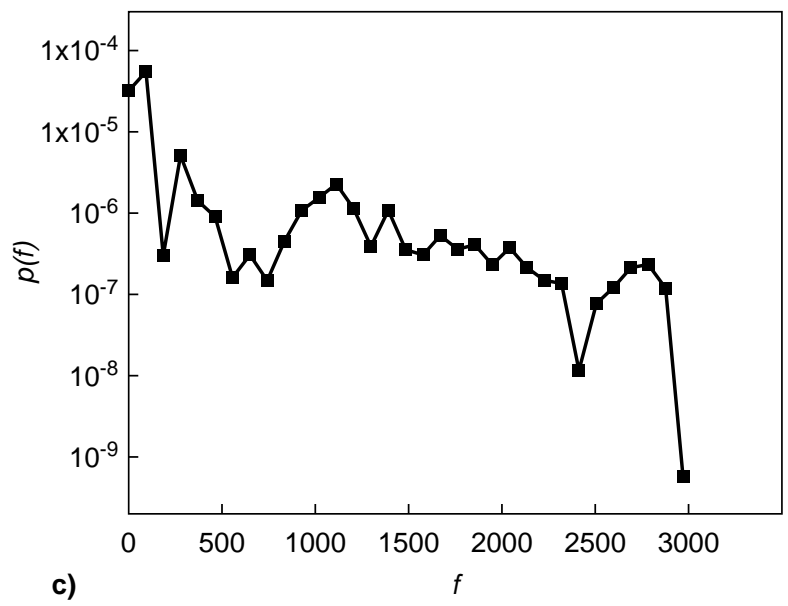

Figure 10

a) temporal evolution; b) autocorrelation function; c) FFT power spectrum of the average solute concentration over a small region (Fig. 9a) of the model pore network D-1 for $P e=3.3$.

Figure 11

a) temporal evolution; b) autocorrelation function; c) FFT power spectrum of the average solute concentration over a small region (Fig. $9 b$ ) of the model pore network D-1 for $P e=4.9$. 
The visualization experimental technique demonstrated that a periodic or quasi-periodic solute dispersion regime is favored by relatively high $P e$ values and relatively low degree of pore-scale heterogeneities, while a chaotic dispersion regime is favored by low $\mathrm{Pe}$ values and high degree of porescale heterogeneities. The instabilities may be caused by absolute permeability perturbations, which strengthen as the spatial heterogeneity of the pore network increases.

The present work is the first experimental demonstration of the onset of buoyancy-driven periodic and chaotic solute dispersion regimes that may arise in a pore network. The high resolution of the experimental technique allows the accurate measurement of the transient fluctuations of the solute concentration at a very wide range of scales. To fully understand the mechanisms and factors that favor the growth of chaotic, periodic, quasi-periodic or fully stable dispersion regimes, a more systematic investigation need to be exerted. Specifically, the duration of the experiments must be extended from several days to several weeks, a great number of datasets has to be collected, and the time series must be employed by using nonlinear methods of state-space analysis. In addition, the mathematical model presented in Section 2 could be used to simulate the single source-solute transport experiments, validate the experimental observations and perform a complete parametric study.

\section{DISCUSSION AND PERSPECTIVES OF APPLICATION}

The dispersive transport of dense plumes in underground reservoirs is one of the most important environmental problems. Two characteristic examples are reported:

- The multispecies leachate plumes that can develop in shallow unconfined aquifers (Zhang and Schwartz, 1995).

- The density variations occurring in groundwater during the injection of liquefied $\mathrm{CO}_{2}$ in it; the aqueous $\mathrm{CO}_{2}$ solution is heavier than pure water even at small concentrations and the identification of the $\mathrm{CO}_{2}$ dispersion regime in the aquifer might be helpful in taking decisions relevant to $\mathrm{CO}_{2}$ sequestration projects.

In flow through a natural porous medium, perturbations or interfacial disturbances are continuously generated because of heterogeneities of the medium. These random perturbations to flow occur over many scales ranging from slight differences in pore geometry to larger heterogeneities on the scale of the problem under consideration. Unresolved questions remain with respect to the manifestation of unstable flows in heterogeneous media. The artificial model pore networks used in the present study enable us to insight into the effect of the pore-scale heterogeneities and flow conditions on the densitydriven contaminant dispersion patterns, and the route from periodic to chaotic solute concentration profiles. Such information may be proved useful in designing landfills or selecting underground saline aquifers for the $\mathrm{CO}_{2}$ storage. Moreover, the experimental data presented here, may be helpful in validating mechanistic pore network or macroscopic simulators of the solute dispersion in homogeneous and heterogeneous porous media. However, longer time series of solute concentration must be collected by increasing by several times the duration of experiments, before a systematic analysis of the chaotic nature of density-driven dispersion regime is clarified quantitatively.

\section{CONCLUSIONS}

Visualization of single source-solute transport experiments performed on three glass-etched pore networks of different geometry reveal the existence of multiple steady-state solute concentration profiles at longer time scale when gravity acts perpendicular to the main flow direction. These dispersion regimes arise from the interaction of forced convective flow, buoyancy-driven free convective flow, and diffusion. Data collected from experiments performed without the action of gravity are fitted to analytical models of the advectiondispersion equation to estimate the longitudinal and transverse dispersivities. The transient evolution of the average solute concentration along with its autocorrelation function and FFT power spectra are employed to get information on the periodic, quasi-periodic or chaotic nature of the fluctuations in the three model pore networks used in the experiments. The most important conclusions are outlined below:

- The estimated values of dispersivities depend strongly on the quantity and quality of experimental datasets, the estimation procedure adopted, and the analytical models used.

- As $P e$ increases, the fluctuations tend to vanish, the mixing zone is reduced, and progressively the dispersion regime is stabilized.

- When the Pe number decreases, and the mixing zone intersects the lateral boundaries of the porous medium, the dispersion regime tends to transit from periodic to chaotic regime.

- Periodic and/or quasi-periodic dispersion regimes are favored by relatively high $P e$ values and small pore-scale heterogeneities.

- Chaotic dispersion regimes are favored by relatively low $P e$ values and strong pore-scale heterogeneities.

- Long time series of solute concentration is required to quantify the periodic/chaotic nature of the fluctuations by using the non-linear state-space analysis.

\section{ACKNOWLEDGEMENTS}

This work was performed under Energy Environment and Sustainable Development (EESD) contract number EVK1CT1999-00013 (project acronym: TRACe-Fracture) supported by the European Commission. 


\section{REFERENCES}

Abarbanel, H.D.I. (1996) Analysis of Observed Chaotic Data, Springer-Verlag, NY.

Addiscott, T.M. and Mizra, N.A. (1998) New Paradigms for Modeling Mass Transfers in Soils. Soil \& Tillage Research, 47, 105-109.

Baker, G.L. and Gollub, G.P. (1996) Chaotic Dynamics - An Introduction, Cambridge University Press, NY.

Basu, A. and Islam, M.R. (1996) Instability in a Combined Heat and Mass Transfer Problem in Porous Media. Chaos, Solitons \& Fractals, 7, 1, 109-123.

Bera, P. and Khalili, A. (2002) Double-Diffusive Natural Convection in an Anisotropic Porous Cavity with Opposing Buoyancy Forces: Multi-Solutions and Oscillations. Int. J. Heat and Mass Transfer, 45, 3205-3222.

Bruggeman, G.A. (1999) Developments in Water Science: Analytical Solutions of Geohydrological Problems, Elsevier, Amsterdam.

Diersch, H.J.G., and Kolditz, O. (2002) Variable-Density Flow and Transport in Porous Media: Approaches and Challenges. Adv. Water Resour., 25, 899-944.

Dimitrova, Z.I. and Vitanov, N.K. (2001) Adaptation and its Impact on the Dynamics of a System of Three Competing Populations. Physica A, 300, 91-115.

Drazin, P.G. (2002) Introduction to Hydrodynamic Stability. Cambridge University Press, UK.

Freedman, V. and Ibaraki, M. (2002) Effects of Chemical Reactions on Density-Dependent Fluid Flow: on the Numerical Formulation and the Development of Instabilities. Adv. Water Resour., 25, 439-453.

Gatica, J.E., Viljoen, H.J. and Hlavacek, V. (1988) Influence of Secondary Flows on the Stability of Chemically Reacting Systems. AIChE J., 34, 2, 209-222.

Grassberger, P. and Procaccia, I. (1984) Dimensions and Entropies of Strange Attractors from a Fluctuating Dynamics Approach. Physica, 13D, 34-54.

Guckenheimer, J. and Holmes, P. (1986) Nonlinear Oscillations, Dynamical Systems, and Bifurcations of Vector Fields, SpringerVerlag, NY.

Johnson, F., Zijerveld, R.C., Schouten, J.C., van den Bleek, C.M. and Leckner, B. (2000) Characterization of Fluidization Regimes by Time-Series Analysis of Pressure Fluctuations. Int. J. Mult. Flow, 26, 663-715.

Liu, H.H., and Dane, J.H. (1997) A Numerical Study on Gravitational Instabilities of Dense Aqueous Phase Plumes in Three-Dimensional Porous Media. J. Hydrology, 194, 126-142.

Maclean, D.J. and Alboussiere, T. (2001) Measurement of Solute Diffusivities. Part I. Analysis of Coupled Solute BuoyancyDriven Convection and Mass Transport. Int. J. Heat and Mass Transfer, 44, 1639-1648.

Perry, R.H. and Chilton, C.H. (1973) Chemical Engineers' Handbook, McGraw-Hill, New York.
Proctor, M.R.E. and Weiss, N.O. (1990) Normal Forms and Chaos in Thermosolutal Convection. Nonlinearity, 3, 619-637.

Sahimi, M. (1995) Flow and Transport in Porous Media and Fractured Rock: From Classical Methods to Modern Approaches, $\mathrm{VCH}$, Weinheim, Germany.

Schincariol, R. (1998) Dispersive Mixing Dynamics of Dense Miscible Plumes: Natural Perturbation Initiation by Local-Scale Heterogeneities. J. Contam. Hydrology, 34, 247-271.

Schoofs, S., Spera, F.J. and Kansen, U. (1999) Chaotic Thermohaline Convection in Low Porosity Hydrothermal Systems. Earth and Planetary Science Letters, 174, 213-229.

Simmons, C.T. and Narayan, K.A. (1997) Mixed Convection Processes Below a Saline Disposal Basin. J. Hydrology, 194, 263-285.

Simmons, C.T., Fenstemaker, T.R. and Sharp, J.M. (2001) Variable-Density Groundwater Flow and Solute Transport in Heterogeneous Porous Media: Approaches, Resolutions and Future Challenges. J. Contam. Hydrology, 52, 245-275.

Takens, F. (1981) Detecting strange attractors in turbulence. In: Proc. Dynamical Systems and Turbulence - Lecture Notes in Mathematics 898, Rand, D.A., Yong, L.S. (Eds.), Springer Verlag, Berlin.

Tsakiroglou, C.D. (2002) Determination of the Transport Properties of Single Fractures with the Aid of Critical Path Analysis. Industrial \& Engineering Chemistry Research, 41, 3462-3472.

Theodoropoulou, M., Karoutsos, V., Kaspiris, C. and Tsakiroglou, C.D. (2003) A New Visualization Technique for the Study of Solute Dispersion in Porous Media Models. J. Hydrology, 274, 176-197.

Tsakiroglou, C.D., Kolonis, G.B., Roumeliotis, T.C. and Payatakes, A.C. (1997) Mercury Penetration and Snap-Off in Lenticular Pores. J. Colloid Interface Science, 193, 259-272.

Tsakiroglou, C.D., Theodoropoulou, M., Karoutsos, V., Papanicolaou, D., Sygouni, V. (2003) Experimental Study of the Immiscible Displecement of Shear-Thinning Fluids in Pore Networks. J. Colloid Interface Science, 267, 217-232.

Turner, J.S. (1973) Buoyancy Effects in Fluids, Cambridge University Press, London.

van Duijn, C.J., Peletier, L.A. and Schotting, R.J. (1998) Brine Transport in Porous Media: Self-Similar Solutions. Adv. Water Resour., 22, 3, 285-297.

Vadasz, P. (1999) Local and Global Transitions to Chaos and Hysteresis in a Porous Layer Heated from Below. Transp. Porous Media, 37, 213-245.

Whitaker, S. (1999) The Method of Volume Averaging, Kluwer, Dordrecht, Netherlands.

Zhang, H. and Schwartz, F.W. (1995). Multispecies Contaminant Plumes in Variable Density Flow Systems. Water Resour. Res., 31, 837-847.

Zhang, H.R., Sorbie, K.S. and Tsibuklis, N.B. (1997) Viscous Fingering in Five-Spot Experimental Porous Media: New Experimental Results and Numerical Simulation. Chem. Eng. Sci., 52, 37-54.

Final manuscript received in November 2004 or distributed for profit or commercial advantage and that copies bear this notice and the full citation on the first page. Copyrights for components of this work owned by others than IFP must be honored. Abstracting with credit is permitted. To copy otherwise, to republish, to post on servers, or to redistribute to lists, requires prior specific permission and/or a fee: Request permission from Documentation, Institut français du pétrole, fax. +33147527078 , or revueogst@ifp.fr. 